\title{
Graphs with Large Disjunctive Total Domination Number
}

\author{
Michael A. Henning $]^{*}$ \\ Viroshan Naicker $\|^{t}$ \\ ${ }^{1}$ Department of Pure and Applied Mathematics, University of Johannesburg, South Africa \\ ${ }^{2}$ Department of Mathematics, Rhodes University, Grahamstown, South Africa \\ received $4^{\text {th }}$ Nov. 2014, accepted $17^{\text {th }}$ Mar. 2015.
}

Let $G$ be a graph with no isolated vertex. In this paper, we study a parameter that is a relaxation of arguably the most important domination parameter, namely the total domination number, $\gamma_{t}(G)$. A set $S$ of vertices in $G$ is a disjunctive total dominating set of $G$ if every vertex is adjacent to a vertex of $S$ or has at least two vertices in $S$ at distance 2 from it. The disjunctive total domination number, $\gamma_{t}^{d}(G)$, is the minimum cardinality of such a set. We observe that $\gamma_{t}^{d}(G) \leq \gamma_{t}(G)$. Let $G$ be a connected graph on $n$ vertices with minimum degree $\delta$. It is known [J. Graph Theory 35 (2000), 21-45] that if $\delta \geq 2$ and $n \geq 11$, then $\gamma_{t}(G) \leq 4 n / 7$. Further [J. Graph Theory 46 (2004), 207-210] if $\delta \geq 3$, then $\gamma_{t}(G) \leq n / 2$. We prove that if $\delta \geq 2$ and $n \geq 8$, then $\gamma_{t}^{d}(G) \leq n / 2$ and we characterize the extremal graphs.

Keywords: Total dominating set; Disjunctive total dominating set

\section{Introduction}

In this paper we continue the study of disjunctive total domination in graphs introduced by the authors in Henning and Naicker (2014). As remarked in Henning and Naicker (2014), a common issue in network design is to minimize the trade-off between resource allocation and redundancy. Key resources are usually expensive and cannot be allocated across an entire network, and, in addition, if there is a possibility of resource failure at a particular node, redundancy and backup requirements then become vital but require extra resources to be allocated. In graph theory, network resource allocation has been addressed, in various guises, by using graphs as a model for the network and searching for vertex subsets which are 'close' to the rest of the graph and satisfy pertinent redundancy criteria.

Domination and, in particular, total domination are well studied topics in the graph theory literature which attempt a solution of this problem (see, for example, Haynes et al. (1998); Henning (2009); Henning and Yeo (2013)). Let $G$ be a graph that serves as a model of a network and let $G$ have vertex set $V$. On the one hand, for purposes of resource allocation and 'closeness', we select a set $D$ of vertices, called a

*Email: mahenning@uj.ac.za

†Email: v.naicker@ru.ac.za

1365-8050 @ 2015 Discrete Mathematics and Theoretical Computer Science (DMTCS), Nancy, France 
dominating set, of $G$ such that every vertex in $V \backslash D$ is adjacent to at least one vertex in $D$. On the other hand, for the purpose of extending the domination problem to include redundancy, we select a set $S$ of vertices, called a total dominating set (abbreviated TD-set) of $G$ such that every vertex in $V$, including those in $S$, is adjacent to at least one vertex in $S$. The cardinality of a minimum total dominating set in $G$ is called the total domination number of $G$ and denoted $\gamma_{t}(G)$. A total dominating set of cardinality $\gamma_{t}(G)$ is called a $\gamma_{t}(G)$-set. It is evident that every $\gamma_{t}(G)$-set is a minimum solution of a resource allocation problem with redundancy for the graph $G$.

Over the last few decades, the scale of networks and the role of graphs as models for networks has changed Bornholdt and Schuster (2003); Chung (2010) and, in practical terms, many existing dominationtype structures are too expensive to implement. The majority of domination-type variants studied to date tend to focus on adding restrictions which in turn raises their implementation costs Haynes et al. (1998). As a result the idea of relaxing conditions on domination-type parameters is appealing. A relaxation of the domination number, called disjunctive domination, was proposed and studied by Goddard et al. Goddard et al. (2014). Inspired by their work, a relaxation of total domination, called disjunctive total domination was proposed in Henning and Naicker (2014). The parameter is defined as follows: Let $G$ be a graph with no isolated vertex. A set $S$ of vertices in $G$ is a disjunctive total dominating set, abbreviated DTD-set, of $G$ if every vertex is adjacent to a vertex of $S$ or has at least two vertices in $S$ at distance 2 from it. For example, the set of eight darkened vertices in the graph $G$ shown in Figure 1 is a DTD-set of $G$. The disjunctive total domination number, $\gamma_{t}^{d}(G)$, is the minimum cardinality of a DTD-set in $G$. A DTD-set of cardinality $\gamma_{t}^{d}(G)$ is called a $\gamma_{t}^{d}(G)$-set.

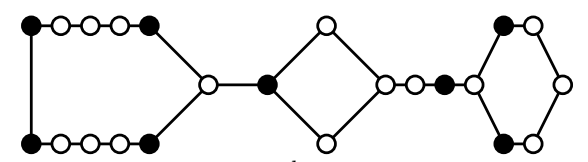

Fig. 1: A graph $G$ with $\gamma_{t}^{d}(G)=8$ and $\gamma_{t}(G)=11$.

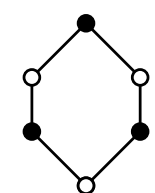

(a)

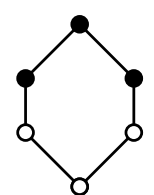

(b)

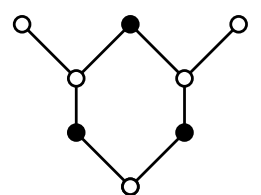

(c)

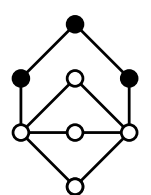

(d)

Fig. 2: Novel features of DTD-sets

There are several interesting features which arise with DTD-sets. Examples of two $\gamma_{t}^{d}(G)$-sets when $G$ is a cycle on six vertices are shown in Figure 2 (a) and Figure 2 b). In each case, the darkened vertices represent a $\gamma_{t}^{d}(G)$-set. A feature of the parameter is, depending on the graph, that a DTD-set may be chosen to be independent Figure 2(a) or connected Figure 2(b). The feature of choosing an independent DTD-set is essentially different from total domination in which independence within the TD-set is, by definition, lost. Further, if new vertices are added to the graph (nodes to a network) these may be added in a way that resources which are already allocated are, in a sense, 'buffered' by their existing neighbors. For example, the graphs in Figure 2(a) and Figure 2(b) have been modified by adding two additional vertices to give Figure 2(c) and Figure 2(d), respectively, while the existing DTD-set is preserved. In each case, 
the vertices need not be adjacent to a vertex in the DTD-set. This is a different feature, again, from total domination in which if a new vertex is joined to the graph by an edge either the existing TD-set must be enlarged and a new resource allocated to the network, or the new vertex has to be joined to a vertex in an existing TD-set which, in turn, invites possible resource overcrowding since new users are joined to the network without new resources being allocated.

Every TD-set is a DTD-set, implying the following observation.

Observation 1 (Henning and Naicker (2014)) For every graph $G$ with no isolated vertex, $\gamma_{t}^{d}(G) \leq$ $\gamma_{t}(G)$.

The known upper bounds on the total domination number of a graph $G$ in terms of its order $n$ and small minimum degree $\delta(G)$ are summarized in Table 1 .

$$
\begin{aligned}
& \hline \hline(G) \geq 1 \Rightarrow \gamma_{t}(G) \leq \frac{2}{3} n \quad \text { if } n \geq 3 \text { and } G \text { is connected } \\
& \delta(G) \geq 2 \Rightarrow \gamma_{t}(G) \leq \frac{4}{7} n \quad \text { if } n \geq 11 \text { and } G \text { is connected } \\
& \delta(G) \geq 3 \Rightarrow \gamma_{t}(G) \leq \frac{1}{2} n
\end{aligned}
$$

Table 1. Upper bounds on the total domination number of a graph $G$.

By Observation 1 and the result of Cockayne et al.(1980) shown in Table 1, if $G$ is a connected graph of order $n \geq 3$, then $\gamma_{t}^{d}(G) \leq 2 n / 3$. The authors recently showed in Henning and Naicker (2014) that this upper bound on the disjunctive total domination number can be improved ever-so-slightly. Further, they characterized the concomitant extremal graphs.

Theorem 2 (Henning and Naicker (2014)) If $G$ is a connected graph of order $n \geq 8$, then $\gamma_{t}^{d}(G) \leq$ $2(n-1) / 3$, and this bound is tight.

In this paper, we show that if we restrict the minimum degree to at least 2 , then the result of Henning (2000) shown in Table 1 on the total domination number can be improved significantly for the disjunctive total domination number. Perhaps surprisingly we show that the upper bound of one-half the order of a graph of Archdeacon et al. (2004); Chvátal and McDiarmid (1992); Tuza (1990) shown in Table 1 on the total domination number is an upper bound on the disjunctive total domination number even if we relax the minimum degree from 3 to 2 . More precisely, we shall prove the following result.

Theorem 3 If $G$ is a connected graph of order $n \geq 13$, then $\gamma_{t}^{d}(G) \leq(n-1) / 2$, and this bound is tight.

Table 2 summarizes the upper bounds on the disjunctive total domination number of a graph $G$ in terms of its order $n$ and minimum degree $\delta(G)$. 


$$
\begin{aligned}
& \delta(G) \geq 1 \Rightarrow \gamma_{t}^{d}(G) \leq \frac{2}{3}(n-1) \quad \text { if } n \geq 8 \text { and } G \text { is connected } \\
& \delta(G) \geq 2 \Rightarrow \gamma_{t}^{d}(G) \leq \frac{1}{2}(n-1) \quad \text { if } n \geq 13 \text { and } G \text { is connected }
\end{aligned}
$$

Table 2: Upper bounds on the disjunctive total domination number of a graph $G$.

\subsection{Notation}

For notation and graph theory terminology, we in general follow Haynes et al. (1998). Specifically, let $G=(V, E)$ be a graph with vertex set $V$ and edge set $E$. We denote the degree of $v$ in $G$ by $d_{G}(v)$. A vertex of degree $k$ is called a degree- $k$ vertex. The maximum (minimum) degree among the vertices of $G$ is denoted by $\Delta(G)(\delta(G)$, respectively). For a set $S \subseteq V$, the subgraph induced by $S$ is denoted by $G[S]$, while the graph obtained from $G$ be removing all vertices in $S$ and their incident edges is denoted by $G-S$. For two vertices $u$ and $v$ in a connected graph $G$, the distance $d_{G}(u, v)$ between $u$ and $v$ is the length of a shortest $(u, v)$-path in $G$. The open neighborhood of a vertex $v$ is the set $N_{G}(v)=\{u \in V \mid u v \in E\}$ and the closed neighborhood of $v$ is $N_{G}[v]=\{v\} \cup N_{G}(v)$. For a set $S \subseteq V$, its open neighborhood is the set $N_{G}(S)=\bigcup_{v \in S} N_{G}(v)$, and its closed neighborhood is the set $N_{G}[S]=N_{G}(S) \cup S$. If the graph $G$ is clear from the context, we simply write $N(v), N[v], N(S), N[S], d(v)$ and $d(u, v)$ rather than $N_{G}(v)$, $N_{G}[v], N_{G}(S), N_{G}[S], d_{G}(v)$, and $d_{G}(u, v)$, respectively.

A cycle and path on $n$ vertices are denoted by $C_{n}$ and $P_{n}$, respectively. For $m \geq 3$ and $n \geq 1$, we denote by $L_{m, n}$ the graph obtained by joining with an edge a vertex in $C_{m}$ to an end-vertex of $P_{n}$. The graph $L_{m, n}$ is called a key.

A 4-subdivision of a graph $G$ is the graph obtained from $G$ by subdividing an edge of $G$ four times. If $e$ is an edge of a graph $G$, we let $G_{e}$ denote the graph obtained from $G$ by subdividing the edge $e$ four times. If two graphs $G$ and $H$ are isomorphic, we write $G \cong H$. Let $G$ be a graph with $\delta(G) \geq 2$. We define a vertex $v$ of $G$ to be large if $d_{G}(v) \geq 3$ and small if $d_{G}(v)=2$. A cycle edge of $G$ is an edge that belongs to a cycle in $G$. We call a vertex, $v$, in a graph $G$ a good-vertex of $G$ if it belongs to some $\gamma_{t}^{d}(G)$-set; otherwise, we call $v$ a bad-vertex of $G$. We call an edge, $e$, in $G$ a good-edge of $G$ if there is a $\gamma_{t}^{d}(G)$-set which contains both vertices incident with $e$; otherwise, we call $e$ a bad-edge.

\subsection{Special Families}

In this section we define several special families of graphs. Let

$$
\mathcal{C}=\left\{C_{3}, C_{4}, C_{5}, C_{6}, C_{7}, C_{8}, C_{9}, C_{11}, C_{12}, C_{13}, C_{17}\right\}
$$

be a family of cycles. A daisy with $k \geq 2$ petals is a connected graph that can be constructed from $k \geq 2$ disjoint cycles by identifying a set of $k$ vertices, one from each cycle into one vertex. If the cycles have lengths $n_{1}, n_{2}, \ldots, n_{k}$, we denote the daisy by $D\left(n_{1}, n_{2}, \ldots, n_{k}\right)$. Let

$$
\mathcal{D}=\{D(3,3), D(4,4), D(3,7)\} .
$$


A dumb-bell is a connected graph on $n=n_{1}+n_{2}+\ell$ vertices that can be constructed by joining a vertex of a cycle $C_{n_{1}}$ to a vertex of a cycle $C_{n_{2}}$ by an edge and subdividing this edge $\ell$ times. If $\ell=0$ we denote the dumb-bell by $D_{b}\left(n_{1}, n_{2}\right)$, and if $\ell \geq 1$ we denote the dumb-bell by $D_{b}\left(n_{1}, n_{2}, \ell\right)$. Let

$$
\begin{aligned}
\mathcal{D}_{b}= & \left\{D_{b}(3,4), D_{b}(3,3,1), D_{b}(4,4), D_{b}(3,4,1), D_{b}(3,3,2), D_{b}(4,7,2),\right. \\
& \left.D_{b}(3,7,3), D_{b}(4,8,1), D_{b}(3,8,2), D_{b}(4,4,5), D_{b}(3,4,6), D_{b}(3,4,7)\right\} .
\end{aligned}
$$

Let $U_{1}, U_{2}, U_{3}$ and $X_{1}, X_{2} \ldots X_{10}$ be the thirteen graphs shown in Figure 3 We define a unit to be a graph that is isomorphic to the graph $U_{i}$ for some $i, 1 \leq i \leq 3$, or the graph $X_{j}$ for some $j, 1 \leq j \leq 10$. The darkened vertex, named $v$, in each unit in Figure 3 we call the link vertex of the unit. For $i=1,2,3$, we call a unit isomorphic to the graph $U_{i}$ a type- $i$ unit. A unit isomorphic to the graph $X_{j}, 1 \leq j \leq 10$, we call a $X_{j}$-unit.
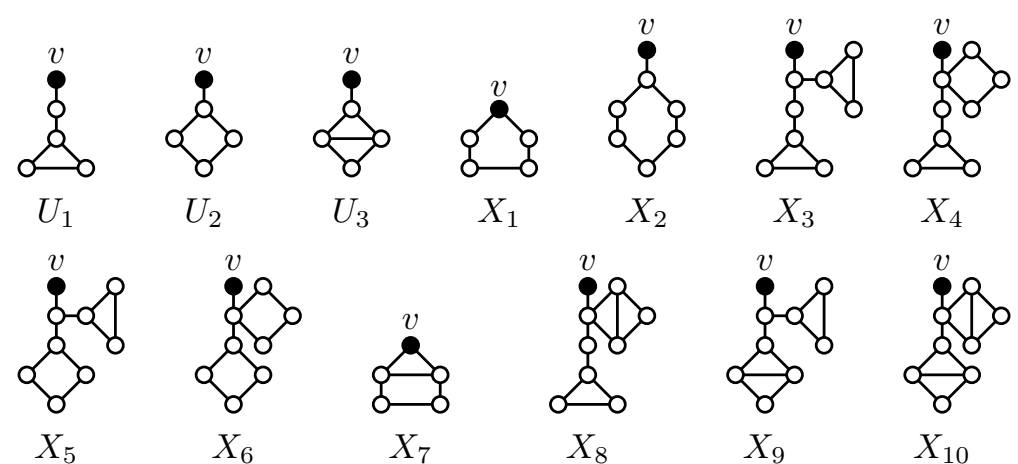

$X_{6}$
Fig. 3: The graphs $U_{1}$

$$
\begin{gathered}
X_{8} \\
U_{3} \text { and } X_{1}, X_{2}, \ldots, X_{10}
\end{gathered}
$$

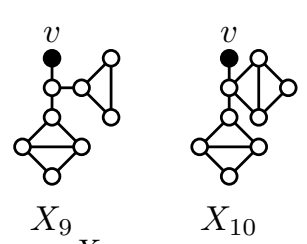

For $n=n_{1}+n_{2} \geq 2$, let $G=G_{0}\left(n_{1}, n_{2}\right)$ be the graph obtained from the disjoint union of $n_{1}$ units of type- 1 and $n_{2}$ units of type- 2 by identifying the $n$ link vertices, one from each unit, into one new vertex which we call the identified vertex of $G$. Let $\mathcal{G}_{0}$ denote the family of all such graphs $G$. For $n=n_{1}+n_{2}+1 \geq 2$ and for $i=1,2, \ldots, 6$, let $G=G_{i}\left(n_{1}, n_{2}\right)$ be the graph obtained from the disjoint union of $n_{1}$ units of type-1, $n_{2}$ units of type-2, and one $X_{i}$-unit by identifying the $n$ link vertices, one from each unit, into one new vertex which we call the identified vertex of $G$. Let $\mathcal{G}_{i}$ denote the family of all such graphs $G$. A graph in each family $\mathcal{G}_{i}, 0 \leq i \leq 6$, is shown in Figure 4, where the identified vertices are indicated in bold. Let $\mathcal{G}=\bigcup_{i=0}^{6} \mathcal{G}_{i}$.

For $n=n_{1}+n_{2}+n_{3} \geq 2$, let $H=H_{0}\left(n_{1}, n_{2}, n_{3}\right)$ be the graph obtained from the disjoint union of $n_{1}$ units of type- $1, n_{2}$ units of type- 2 and $n_{3}$ units of type- 3 by identifying the $n$ link vertices, one from each unit, into one new vertex which we call the identified vertex of $H$. Let $\mathcal{H}_{0}$ denote the family of all such graphs $H$. For $n=n_{1}+n_{2}+n_{3}+1 \geq 2$ and for $i=1,2, \ldots, 10$, let $H=H_{i}\left(n_{1}, n_{2}, n_{3}\right)$ be the graph obtained from the disjoint union of $n_{1}$ units of type- $1, n_{2}$ units of type- $2, n_{3}$ units of type- 3 , and one $X_{i}$-unit by identifying the $n$ link vertices, one from each unit, into one new vertex which we call the identified vertex of $H$. Let $\mathcal{H}_{i}$ denote the family of all such graphs $H$. Let $\mathcal{H}=\bigcup_{i=0}^{10} \mathcal{H}_{i}$. We observe that $\mathcal{G}$ is a subfamily of $\mathcal{H}$; that is, $\mathcal{G} \subset \mathcal{H}$. Let $\mathcal{G}_{b}$ be the subfamily of $\mathcal{G}$ consisting of all dumb-bells. We observe that

$$
\mathcal{G}_{b}=\left\{G_{0}(2,0), G_{0}(1,1), G_{0}(0,2), G_{1}(1,0), G_{1}(0,1), G_{2}(1,0), G_{2}(0,1)\right\}
$$



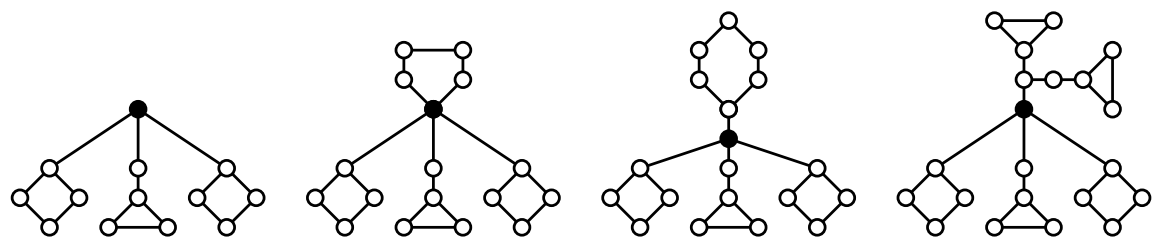

\section{$G_{0}(1,2) \in \mathcal{G}_{0}$}

$G_{1}(1,2) \in \mathcal{G}_{1}$

$G_{2}(1,2) \in \mathcal{G}_{2}$

$G_{3}(1,2) \in \mathcal{G}_{3}$
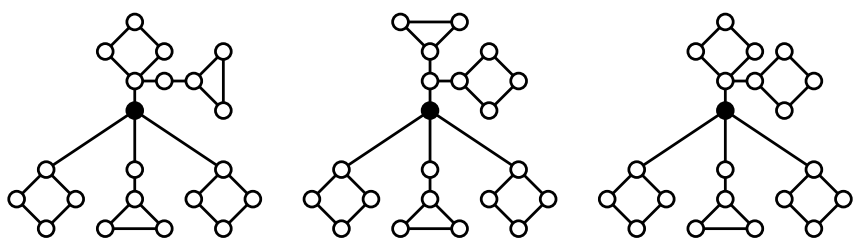

$$
G_{4}(1,2) \in \mathcal{G}_{4}
$$

$G_{5}(1,2) \in \mathcal{G}_{5}$

$G_{6}(1,2) \in \mathcal{G}_{6}$

Fig. 4: Graphs in the family $\mathcal{G}$.

Let $\mathcal{F}$ be the family of five graphs shown in Figure 5 and let $\mathcal{B}=\left\{B_{1}, B_{2}, \ldots, B_{11}\right\}$ be the family of eleven graphs shown in Figure 6, where the special vertices (to be defined later in Section 3.2) of each graph are darkened.

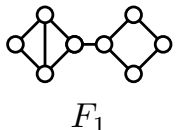

$F_{1}$

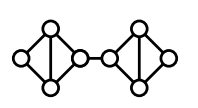

$F_{2}$

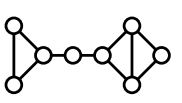

$F_{3}$

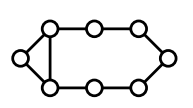

$F_{4}$

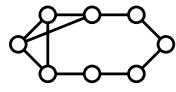

$F_{5}$

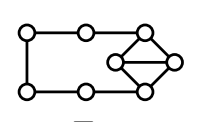

$F_{6}$

Fig. 5: The family, $\mathcal{F}$, of graphs.

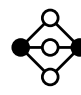

$B_{1}$

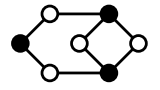

$B_{2}$

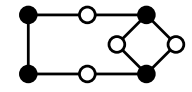

$B_{3}$

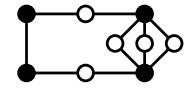

$B_{4}$

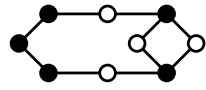

$B_{5}$

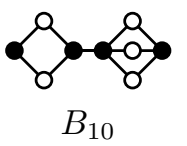

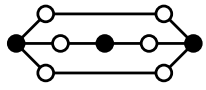

$B_{6}$

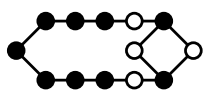

$B_{11}$

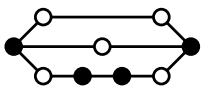

$B_{7}$

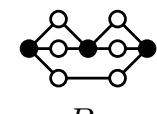

$B_{8}$

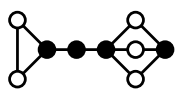

$B_{9}$

Fig. 6: The family, $\mathcal{B}$, of graphs.

\section{Main Results}

Our aim in this paper is to improve the upper bound of Theorem 2 on the disjunctive total domination number of a connected graph when we impose a density condition by restricting the minimum degree to be at least 2. In this case, we show that the result of Henning (2000) given in Table 1 on the total domination number can be improved significantly for the disjunctive total domination number. Several 
authors (see Archdeacon et al. (2004); Chvátal and McDiarmid (1992); Tuza (1990)) showed that if $G$ is a graph of order $n$ with $\delta(G) \geq 3$, then $\gamma_{t}(G) \leq n / 2$. We prove that if we relax the minimum degree condition from $\delta(G) \geq 3$ to $\delta(G) \geq 2$, then $n / 2$ is an upper bound on the disjunctive total domination number $\gamma_{t}^{d}(G)$, provided $n \geq 8$. More precisely, we prove the following result. A proof of Theorem 4 is given in Section 4.2 .

Theorem 4 Let $G$ be a connected graph of order $n \geq 8$ with $\delta(G) \geq 2$. Then, $\gamma_{t}^{d}(G) \leq n / 2$ with equality if and only if $G \in\left\{C_{8}, C_{12}, B_{3}, D_{b}(4,4), D_{b}(3,4,1), D_{b}(3,3,2)\right\} \cup \mathcal{F}$.

The connected graphs with minimum degree at least 2 and order at least 18 that have maximum possible disjunctive total domination number are characterized in the following result, a proof of which is given in Section 4.3 ,

Theorem 5 Let $G$ be a connected graph of order $n \geq 18$ with $\delta(G) \geq 2$. Then, $\gamma_{t}^{d}(G) \leq(n-1) / 2$ with equality if and only if $G \in \mathcal{H}$.

Since the graphs that achieve equality in the upper bound of Theorem 4 all have order at most 12 , we remark that Theorem 3 is an immediate consequence of Theorem 4 and Theorem 5

\section{Preliminary Results}

Before presenting a proof of our main results, we first establish some preliminary results. We omit the proofs of these preliminary results which are straightforward, albeit tedious, to check ${ }_{[\text {(i) }}$

Observation 6 Let $G$ be a graph with no isolated vertex and let $F$ be a 4-subdivision of $G$. Then, $\gamma_{t}^{d}(F) \leq \gamma_{t}^{d}(G)+2$.

The disjunctive total domination number of a cycle $C_{n}$ on $n$ vertices is established in Henning and Naicker (2014).

Proposition 1 (Henning and Naicker (2014)) For $n \geq 3, \gamma_{t}^{d}\left(C_{n}\right)=2 n / 5$ if $n \equiv 0(\bmod 5)$ and $\gamma_{t}^{d}\left(C_{n}\right)=\lceil 2(n+1) / 5\rceil$ otherwise.

The daisies and dumb-bells with large disjunctive total domination number are characterized in Proposition 2 and Proposition 3 , respectively.

Proposition 2 If $G$ is a daisy of order $n$, then $\gamma_{t}^{d}(G) \leq(n-1) / 2$. Furthermore, $\gamma_{t}^{d}(G)=(n-1) / 2$ if and only if $G \in \mathcal{D}$.

Proposition 3 If $G$ is a dumb-bell of order $n$, then $\gamma_{t}^{d}(G) \leq n / 2$. Furthermore, $\gamma_{t}^{d}(G) \geq(n-1) / 2$ if and only if $G \in \mathcal{D}_{b} \cup \mathcal{G}_{b}$.

The disjunctive total domination number of graphs in the family $\mathcal{B} \cup \mathcal{C} \cup \mathcal{D} \cup \mathcal{D}_{b} \cup \mathcal{G}$ is given by Observation 7

Observation 7 Let $G \in \mathcal{B} \cup \mathcal{C} \cup \mathcal{D} \cup \mathcal{D}_{b} \cup \mathcal{G}$ have order $n$. Then, $G$ is a connected graph with $\delta(G)=2$. Further, $\gamma_{t}^{d}(G)=(n+1) / 2$ if $G \in\left\{C_{3}, C_{7}\right\}, \gamma_{t}^{d}(G)=n / 2$ if $G \in\left\{C_{4}, C_{6}, C_{8}, C_{12}, B_{3}, D_{b}(4,4)\right.$, $\left.D_{b}(3,4,1), D_{b}(3,3,2)\right\}$ and $\gamma_{t}^{d}(G)=(n-1) / 2$ otherwise.

(i) Details of proofs of the preliminary results can be found in Henning and Naicker 


\section{1 $\frac{1}{2}$-Minimal Graphs}

In order to prove our two main results, we study so-called $\frac{1}{2}$-minimal graphs. A graph $G$ is $\frac{1}{2}$-minimal graph if $G$ is edge-minimal with respect to the following three conditions: (i) $\delta(G) \geq 2$, (ii) $G$ is connected, and (iii) $\gamma_{t}^{d}(G) \geq(n-1) / 2$, where $n$ is the order of $G$. If $G$ is edge-minimal with respect to conditions (i) and (ii) but does not necessarily satisfy condition (iii) we refer to $G$ as an edge-minimal graph. Thus if $G$ is an edge-minimal graph and $e \in E(G)$, then $\delta(G-e)=1$ or $G-e$ is disconnected. It is evident that, an edge-minimal graph which satisfies condition (iii) is a $\frac{1}{2}$-minimal graph.

As an immediate consequence of Proposition 1, 2, and 3, we obtain a characterization of the $\frac{1}{2}$-minimal graphs that are cycles, daisies and dumb-bells.

Corollary 8 Let $G$ be a $\frac{1}{2}$-minimal graph. Then the following holds.

(a) $G$ is a cycle if and only if $G \in \mathcal{C}$.

(b) $G$ is a daisy if and only if $G \in \mathcal{D}$.

(c) $G$ is a dumb-bell if and only if $G \in \mathcal{D}_{b} \cup \mathcal{G}_{b}$.

We next establish properties of graphs in the family $\mathcal{B} \cup \mathcal{C} \cup \mathcal{D} \cup \mathcal{D}_{b} \cup \mathcal{G}$.

Observation 9 Each graph in $\mathcal{B} \cup \mathcal{C} \cup \mathcal{D} \cup \mathcal{D}_{b} \cup \mathcal{G}$ is a $\frac{1}{2}$-minimal graph.

Recall that an edge $e$ in a graph $G$ is a good-edge of $G$ if there is a $\gamma_{t}^{d}(G)$-set which contains both ends of $e$; otherwise, it is a bad-edge.

Observation 10 Let $G \in \mathcal{B} \cup \mathcal{C} \cup \mathcal{D} \cup \mathcal{D}_{b} \cup \mathcal{G}$. Then every edge of $G$ is a good-edge of $G$ unless $G \in\left\{D(4,4), D_{b}(3,4), D_{b}(3,3,1)\right\} \cup \mathcal{G} \backslash \mathcal{G}_{1}$. Furthermore, if $e$ is a bad-edge of $G$, then the following holds.

(a) If $G=D(4,4)$, then $e$ is not incident with the vertex of degree 4 in $G$.

(b) If $G=D_{b}(3,4)$, then $e$ is not incident with a vertex of degree 3 in $G$.

(c) If $G=D_{b}(3,3,1)$, then $e$ is a cycle edge of $G$.

(d) If $G \in \mathcal{G} \backslash \mathcal{G}_{1}$, then $e$ is incident with the identified vertex of $G$.

Recall that a vertex $v$ in a graph $G$ is a good-vertex of $G$ if it belongs to some $\gamma_{t}^{d}(G)$-set; otherwise, it is a bad-vertex of $G$.

Observation 11 Let $G \in \mathcal{B} \cup \mathcal{C} \cup \mathcal{D} \cup \mathcal{D}_{b} \cup \mathcal{G}$. Then every vertex of $G$ is a good-vertex of $G$ unless $G \in\left\{D(4,4), D_{b}(3,4), D_{b}(3,3,1)\right\} \cup \mathcal{G}_{0}$. Furthermore, if $v$ is a bad-vertex of $G$, then the following holds.

(a) If $G \in \mathcal{G}_{0}$, then $v$ is the identified vertex of $G$.

(b) If $G \in\left\{D(4,4), D_{b}(3,4), D_{b}(3,3,1)\right\}$, then $v$ is a vertex at distance 2 from the central vertex of $G$.

The following observation establishes a property of good-graphs which are not dumb-bells.

Observation 12 If $G \in \mathcal{G}$ and $v$ is the identified vertex of $G$, then there is a $\gamma_{t}^{d}(G)$-set, $S$, such that $v \notin S$ and $N(v) \subset S$. Further if $G \notin \mathcal{G}_{1}$, then $S$ can be chosen to totally dominate $N(v)$.

The following observation characterizes the $\frac{1}{2}$-minimal graphs of small order.

Observation 13 If $G$ is a $\frac{1}{2}$-minimal graph of order $n, 3 \leq n \leq 7$, then $G \in\left\{B_{1}, B_{2}, C_{3}, C_{4}, C_{5}, C_{6}\right.$, $\left.D(3,3), D(4,4), D_{b}(3,4), D_{b}(3,3,1)\right\} \subset \mathcal{B} \cup \mathcal{C} \cup \mathcal{D} \cup \mathcal{D}_{b}$. 


\subsection{Near Disjunctive Total Dominating Sets}

Let $G$ be a graph and let $v$ be a vertex of $G$. We denote the graph obtained from $G$ by adding a new vertex $v^{\prime}$ and adding the pendant edge $v v^{\prime}$ by $G^{v}$. We define a near-disjunctive total dominating set, abbreviated NDTD-set, of $G^{v}$ to be a set $S$ of vertices in $G^{v}$ such that $v^{\prime} \in S$ and every vertex in $V(G)$ is DT-dominated by the set $S$ in $G^{v}$. We observe that if $S$ is a NDTD-set of $G^{v}$, then possibly the vertex $v^{\prime}$ may not be DT-dominated by $S$. The near-disjunctive total domination number, $\gamma_{n t}^{d}\left(G^{v}\right)$, of $G^{v}$ is the minimum cardinality of a NDTD-set in $G^{v}$. A NDTD-set of cardinality $\gamma_{n t}^{d}\left(G^{v}\right)$ is called a $\gamma_{n t}^{d}\left(G^{v}\right)$-set.

We say that a vertex $v$ of an edge-minimal graph $G$ is special if for every edge $e=u v$ incident with $v$ we have that $G-e$ is disconnected or $d_{G}(u)=2$. In particular, we note that if $v$ is a special-vertex of $G$, then $\delta(G-v)=1$ or $G-v$ is disconnected.

We present next two observations that establish useful properties of graphs that belong to the family $\mathcal{B} \cup \mathcal{C} \cup \mathcal{D} \cup \mathcal{D}_{b} \cup \mathcal{G}$.

Observation 14 Let $G \in \mathcal{B} \cup \mathcal{C} \cup \mathcal{D} \cup \mathcal{D}_{b} \cup \mathcal{G}$ and let $v$ be a special vertex of $G$. Then, $\gamma_{n t}^{d}\left(G^{v}\right) \leq \gamma_{t}^{d}(G)$, unless one of the following four conditions hold.

(a) $G \in\left\{C_{4}, C_{5}\right\}$.

(b) $G=B_{1}$ and $v$ has degree 3 in $G$.

(c) $G \in \mathcal{G}$ and $v$ is the identified vertex of $G$.

(d) $G \in \mathcal{G}_{0}$ and $v$ is a neighbor of the identified vertex of $G$.

Observation 15 Let $G \in \mathcal{B} \cup \mathcal{C} \cup \mathcal{D} \cup \mathcal{D}_{b} \cup \mathcal{G}$ and let $v$ be a special vertex of $G$. Let $v^{\prime}$ be the vertex added to $G$ when constructing $G^{v}$. If $\gamma_{n t}^{d}\left(G^{v}\right) \leq \gamma_{t}^{d}(G)$, then there exists a $\gamma_{n t}^{d}\left(G^{v}\right)$-set that DT-dominates $v^{\prime}$ unless one of the following two conditions hold.

(a) $G=D(4,4)$ and $v$ is at distance 2 from the vertex of degree 4 in $G$.

(b) $G \in\left\{D_{b}(3,4), D_{b}(3,3,1)\right\}, d_{G}(v)=3$, and $v$ belongs to a 3 -cycle in $G$.

\section{Proof of Main Results}

In this section, we present a proof of our main results, namely Theorem 4 and Theorem 5 . We begin with a characterization of $\frac{1}{2}$-minimal graphs.

\subsection{A Characterization of $\frac{1}{2}$-Minimal Graphs}

A key result to enable us to prove our main results is the following characterization of $\frac{1}{2}$-minimal graphs.

Theorem 16 A graph $G$ is $\frac{1}{2}$-minimal if and only if $G \in \mathcal{B} \cup \mathcal{C} \cup \mathcal{D} \cup \mathcal{D}_{b} \cup \mathcal{G}$.

Proof of Theorem 19. The sufficiency follows from Observation 9 . To prove the necessity, we proceed by induction on the order $n \geq 3$ of a $\frac{1}{2}$-minimal graph. By Observation 13 the result is true for $n \leq 7$. Suppose $n \geq 8$, and assume that the result is true for all $\frac{1}{2}$-minimal graphs $G$ of order $n^{\prime}$, where $3 \leq n^{\prime}<$ $n$. Let $G=(V, E)$ be a $\frac{1}{2}$-minimal graph of order $n$. We first present two useful observations. If $e$ is an edge of $G$, then $\gamma_{t}^{d}(G-e) \geq \gamma_{t}^{d}(G)$. Hence, by the minimality of $G$, we have the following observation.

Observation 17 If $e \in E$, then either $e$ is a bridge of $G$ or $\delta(G-e)=1$.

Since the disjunctive total domination number cannot decrease if edges are removed, the next observation follows as a consequence of the inductive hypothesis. 
Observation 18 If $G^{\prime}$ is a connected subgraph of $G$ of order $n^{\prime}<n$ with $\delta\left(G^{\prime}\right) \geq 2$, then either $G^{\prime} \in$ $\mathcal{B} \cup \mathcal{C} \cup \mathcal{D} \cup \mathcal{D}_{b} \cup \mathcal{G}$ or $\gamma_{t}^{d}\left(G^{\prime}\right) \leq(n-2) / 2$.

We now return to the proof of Theorem 16 Suppose $G=C_{n}$ (and still $n \geq 8$ ). Then, by Corollary 8 . $G \in \mathcal{C}$. Hence we may assume $G$ is not a cycle. Let $\mathcal{L}$ be the set of all large vertices of $G$ and let $\mathcal{S}$ be the set of small vertices in $G$, i.e., $\mathcal{L}=\left\{v \in V \mid d_{G}(v) \geq 3\right\}$ and $\mathcal{S}=\left\{v \in V \mid d_{G}(v)=2\right\}$. Since $G$ is not a cycle, $|\mathcal{L}| \geq 1$. If $|\mathcal{L}|=1$, then $G$ is a daisy, and by Corollary 8 (b), $G \in \mathcal{D}$. Hence, we may assume $|\mathcal{L}| \geq 2$. Further, if $|\mathcal{L}|=2$ and $G$ is a dumb-bell, then by Corollary 8 (c), $G \in \mathcal{D}_{b} \cup \mathcal{G}_{b}$. Hence, we may assume that if $|\mathcal{L}|=2$, then $G$ is not a dumb-bell.

Let $C$ be any component of $G-\mathcal{L}$; it is a path. If $C$ has only one vertex, or has at least two vertices but the ends of $C$ are adjacent in $G$ to different large vertices, then we say that $C$ is a 2-path. Otherwise we say that $C$ is a 2 -handle.

Lemma 19 If $\mathcal{L}$ is not an independent set, then $G \in\left\{B_{10}, D_{b}(3,4), D_{b}(4,4)\right\} \cup \mathcal{G}$.

Proof of Lemma 19. Suppose that $\mathcal{L}$ is not an independent set. Let $u$ and $v$ be two adjacent vertices in $\mathcal{L}$ and let $e=u v$. By Observation 17, $e$ is a bridge. Let $G_{1}=\left(V_{1}, E_{1}\right)$ and $G_{2}=\left(V_{2}, E_{2}\right)$ be the two components of $G-e$, where $u \in V_{1}$. For $i=1,2$, let $\left|V_{i}\right|=n_{i}$, and so $n=n_{1}+n_{2}$. Since $u, v \in \mathcal{L}$ in $G$, we note that $\delta\left(G_{1}\right) \geq 2$ and $\delta\left(G_{2}\right) \geq 2$. Hence, by Observation 18 , for $i=1,2$, either $\gamma_{t}^{d}\left(G_{i}\right) \leq\left(n_{i}-2\right) / 2$ or $G_{i} \in \mathcal{B} \cup \mathcal{C} \cup \mathcal{D} \cup \mathcal{D}_{b} \cup \mathcal{G}$. If $\gamma_{t}^{d}\left(G_{i}\right) \leq\left(n_{i}-1\right) / 2$ for $i=1,2$, then, $\gamma_{t}^{d}(G) \leq\left(n_{1}-1\right) / 2+\left(n_{2}-1\right) / 2=(n-2) / 2$, a contradiction. Hence we may assume that $\gamma_{t}^{d}\left(G_{1}\right) \geq n_{1} / 2$. By Observation 7 , if $\gamma_{t}^{d}\left(G_{1}\right)>n_{1} / 2$, then $G_{1} \in\left\{C_{3}, C_{7}\right\}$ and if $\gamma_{t}^{d}(G)=n_{1} / 2$, then $G_{1} \in\left\{C_{4}, C_{6}, C_{8}, C_{12}\right\} \cup\left\{B_{3}, D_{b}(4,4), D_{b}(3,4,1), D_{b}(3,3,2)\right\}$.

Let $N_{v}$ denote the set of neighbors of $v$ in $G_{2}$, and so $N_{v}=N_{G}(v) \backslash\{u\}$. If $V\left(G_{2}\right)=N_{v} \cup\{v\}$, then $\{u, v\}$ is a DTD-set of $G$, and so recalling that $n \geq 8, \gamma_{t}^{d}(G)=2<(n-2) / 2$, a contradiction. Hence, $V\left(G_{2}\right) \neq N_{v} \cup\{v\}$. We proceed further with the following series of claims.

Claim 1 If $G_{1}=C_{3}$, then $G \in \mathcal{G}_{3} \cup \mathcal{G}_{5}$.

Proof of Claim 1. Suppose that $G_{1}=C_{3}$. Since $G$ is not a dumb-bell, the graph $G_{2}$ is not a cycle. The vertex $v$ has at least two neighbors in $G_{2}$, and so $\left|N_{v}\right| \geq 2$. Further, every vertex in $V\left(G_{2}\right) \backslash N[v]$ has degree at least 2 in $G_{2}-v$.

We claim that the set $N_{v}$ is an independent set in $G$. Suppose, to the contrary, that $x_{1}$ and $x_{2}$ are two adjacent vertices in $N_{v}$. If $x_{1}$ and $x_{2}$ are both large vertices, let $f=x_{1} x_{2}$, while if $x_{1}$ is a large vertex and $x_{2}$ is a small vertex, let $f=v x_{1}$. In both cases, $G-f$ is connected and $\delta(G-f) \geq 2$, contradicting the edge-minimality of $G$. Hence, both $x_{1}$ and $x_{2}$ are small vertices. Thus, $G$ contains a 2-handle $C$ with $|C|=2$ and with both ends of $C$ adjacent to $v$. Since $V\left(G_{2}\right) \neq N_{v} \cup\{v\}$, we observe that $\left|N_{v}\right| \geq 3$ and $d_{G}(v) \geq 4$. Let $H=G-\left\{x_{1}, x_{2}\right\}$. The degree of each vertex in $H$ different from $v$ remains unchanged from its degree in $G$, and so $H$ is a connected graph with $\delta(H) \geq 2$. Further, $H$ is edge-minimal. Let $H$ have order $n_{H}=n-2$. By Observation 18. $H \in \mathcal{B} \cup \mathcal{D} \cup \mathcal{D}_{b} \cup \mathcal{G}$ or $\gamma_{t}^{d}(H) \leq\left(n_{H}-2\right) / 2$.

Suppose $\gamma_{t}^{d}(H) \leq\left(n_{H}-2\right) / 2$. Every $\gamma_{t}^{d}(H)$-set can be extended to a DTD-set of $G$ by adding to it the vertex $v$, implying that $\gamma_{t}^{d}(G) \leq \gamma_{t}^{d}(H)+1 \leq\left(n_{H}-2\right) / 2+1=(n-2) / 2$, a contradiction. Hence, $H \in \mathcal{B} \cup \mathcal{D} \cup \mathcal{D}_{b} \cup \mathcal{G}$. Since $G_{1}$ is a subgraph of $H$, we note that $H$ is not a cycle. Therefore by Observation 7. $\gamma_{t}^{d}(H) \leq n_{H} / 2$. If $v$ is a good-vertex of $H$, then a $\gamma_{t}^{d}(H)$-set that contains $v$ is a DTD-set of $G$, implying that $\gamma_{t}^{d}(G) \leq \gamma_{t}^{d}(H) \leq n_{H} / 2=(n-2) / 2$, a contradiction. Therefore, $v$ is a bad-vertex of $H$. By Observation 7 , either $H \in\left\{D(4,4), D_{b}(3,4), D_{b}(3,3,1)\right\}$ and $v$ is a vertex at distance 2 from 
the central vertex of $H$ or $H \in \mathcal{G}_{0}$ and $v$ is the identified vertex of $H$. In both cases, $G_{1}$ is not a subgraph of $H$, a contradiction. Therefore, set $N_{v}$ is an independent set in $G$.

We claim next that if $N_{v}$ contains two small vertices in $G$, then these two vertices have only $v$ as their common neighbor. Suppose, to the contrary, that $w_{1}$ and $w_{2}$ are two small vertices (of degree 2) in $N_{v}$ and that $N\left(w_{1}\right)=N\left(w_{2}\right)=\{v, x\}$. Suppose that $d_{G}(x)=2$. In this case we consider the connected graph $H=G-\left\{w_{1}, w_{2}, x\right\}$. Let $H$ have order $n_{H}=n-3$. Since $G$ is not a dumb-bell, $d_{H}(v) \geq 2$ and $H$ is edge-minimal. Further since $H$ is not a cycle, $\gamma_{t}^{d}(H) \leq n_{H} / 2$. Since $G_{1}$ is a subgraph of $H$, we can choose a $\gamma_{t}^{d}(H)$-set to contain $u$ and $v$ or to contain $u$ and at least two vertices in $N_{v}$. In both cases, such a $\gamma_{t}^{d}(H)$-set can be extended to a DTD-set of $G$ by adding to it the vertex $w_{1}$, implying that $\gamma_{t}^{d}(G) \leq \gamma_{t}^{d}(H)+1$. If $\gamma_{t}^{d}(H) \leq\left(n_{H}-1\right) / 2$, then $\gamma_{t}^{d}(G) \leq(n-2) / 2$, a contradiction. Hence, $\gamma_{t}^{d}(H)=n_{H} / 2$, implying by Observation 7 and our earlier observations, that $H=D_{b}(3,4,1)$ or $\left.H=D_{b}(3,3,2)\right\}$. In both cases, $\gamma_{t}^{d}(G) \leq 4=(n-2) / 2$, a contradiction. Hence, $d_{G}(x) \geq 3$.

We now consider the graph $H=G-w_{1}$. The degree of each vertex in $H$ different from $v$ and $x$ remains unchanged from its degree in $G$. Further, $d_{H}(v) \geq 2$ and $d_{H}(x) \geq 2$, and so $H$ is a connected graph with $\delta(H) \geq 2$, implying that $H$ is edge-minimal. Let $H$ have order $n_{H}=n-1$. Since $G_{1}$ is a subgraph of $H$, we can choose a $\gamma_{t}^{d}(H)$-set to contain $u$ and $v$ or to contain $u$ and at least two vertices in $N_{v}$. Such a $\gamma_{t}^{d}(H)$-set is a DTD-set of $G$, implying that $\gamma_{t}^{d}(G) \leq \gamma_{t}^{d}(H)$. Since $H$ is not a cycle, $\gamma_{t}^{d}(H) \leq n_{H} / 2$. If $\gamma_{t}^{d}(H) \leq\left(n_{H}-1\right) / 2$, then $\gamma_{t}^{d}(G) \leq(n-2) / 2$, a contradiction. Hence, $\gamma_{t}^{d}(H)=n_{H} / 2$, implying by Observation 7 and our earlier observations, that $H=D_{b}(3,3,2)$. But then $\left\{u, w_{1}, w_{2}\right\}$ is a DTD-set in $G$, and so $\gamma_{t}^{d}(G) \leq 3=(n-3) / 2$, a contradiction. Therefore, if $N_{v}$ contains two small vertices in $G$, then these two vertices have only $v$ as their common neighbor.

Among all vertices in $N_{v}$, let $w$ be one of minimum degree. We note that $d_{G_{2}-v}(w) \geq 1$. Let $G_{2}^{*}$ be the graph obtained from $G_{2}-v$ by adding as few edges as possible joining $w$ to vertices in $N_{v}$ so that the resulting graph is connected and has minimum degree at least 2 . We note that by our earlier observations, and by the fact that $G$ is edge-minimal, the graph $G_{2}^{*}$ is edge-minimal. By the inductive hypothesis, $G_{2}^{*} \in \mathcal{B} \cup \mathcal{C} \cup \mathcal{D} \cup \mathcal{D}_{b} \cup \mathcal{G}$ or $\gamma_{t}^{d}\left(G_{2}^{*}\right) \leq\left(n_{2}^{*}-2\right) / 2$. Let $S^{*}$ be a $\gamma_{t}^{d}\left(G_{2}^{*}\right)$-set.

We claim next that $G_{2}^{*} \in \mathcal{B} \cup \mathcal{C} \cup \mathcal{D} \cup \mathcal{D}_{b} \cup \mathcal{G}$. Suppose $\gamma_{t}^{d}\left(G_{2}^{*}\right) \leq\left(n_{2}^{*}-2\right) / 2=(n-6) / 2$. If $w \in S^{*}$ or if $S^{*} \cap N_{v}=\emptyset$, let $S=S^{*} \cup\{u, v\}$. If $w \notin S^{*}$ and $\left|S^{*} \cap N_{v}\right| \geq 1$, let $S=S^{*} \cup\{u, w\}$. We show that $S$ is a DTD-set in $G$. Suppose that there is a vertex $x$ in $G$ that is not DT-dominated by the set $S$. Then, $x$ has no neighbor in $S$ and is at distance 2 from at most one vertex of $S$ in $G$. If $x=u$, then $v \notin S$, implying that $S=S^{*} \cup\{u, w\}$. In this case, $\left|S \cap N_{v}\right| \geq 2$ and therefore $x$ is at distance 2 from at least two vertices of $S$, a contradiction. Hence, $x \neq u$. Since $u \in S$, we have that $x \notin N(u)$, and so $x \in V\left(G_{2}^{*}\right)$. Suppose $x \in N_{v}$. Then, $v \notin S$, implying that $w \in S, w \notin S^{*}$ and $\left|S^{*} \cap N_{v}\right| \geq 1$. Let $w^{*} \in S^{*} \cap N_{v}$. If $x \neq w$, then $x$ is at distance 2 from both $u$ and $w$. If $x=w$, then $x$ is at distance 2 from both $u$ and $w^{*}$. In both cases, $x$ is at distance 2 from at least two vertices of $S$, a contradiction. Hence, $x \notin N_{v}$. Thus, the neighbors of $x$ in $G$ and $G_{2}^{*}$ are the same. Since $S^{*} \subset S$ and $x$ has no neighbor in $S$, the vertex $x$ has no neighbor in $S^{*}$. However, $S^{*}$ is a DTD-set of $G_{2}^{*}$, implying that $x$ is at distance 2 from at least two vertices of $S^{*}$ in $G_{2}^{*}$. Since $x$ is at distance 2 from at most one vertex of $S$ in $G$ and since $S^{*} \subset S$, there is a vertex $x^{*} \in S^{*}$ at distance 2 from $x$ in $G_{2}^{*}$ but at distance greater than 2 from $x$ in $G$. This is only possible if $w$ is the only common neighbor of $x$ and $x^{*}$ in $G_{2}^{*}$ and if $w x^{*}$ was an edge added to $G_{2}-v$ when forming $G_{2}^{*}$. Therefore, $x^{*} \in S^{*} \cap N_{v}$ and $x^{*} \neq w$. In particular, we note that $w \notin S^{*}$ and $\left|S^{*} \cap N_{v}\right| \geq 1$. But then $w \in S$, implying that $x$ has a neighbor in $S$, a contradiction. Therefore, $S$ is a DTD-set in $G$. Thus, $\gamma_{t}^{d}(G) \leq|S|=\left|S^{*}\right|+2=\gamma_{t}^{d}\left(G_{2}^{*}\right)+2 \leq(n-6) / 2+2=(n-2) / 2$, a contradiction. Hence, $G_{2}^{*} \in \mathcal{B} \cup \mathcal{C} \cup \mathcal{D} \cup \mathcal{D}_{b} \cup \mathcal{G}$. Therefore, $G_{2}^{*} \in \mathcal{B} \cup \mathcal{C} \cup \mathcal{D} \cup \mathcal{D}_{b} \cup \mathcal{G}$. 
Suppose that $d_{G}(w)=2$. Then, $d_{G_{2}-v}(w)=1$ and therefore at least one new edge incident with $w$ was added to $G_{2}-v$ to guarantee that $\delta\left(G_{2}^{*}\right) \geq 2$. On the other hand, suppose that $d_{G}(w) \geq 3$. By our choice of $w$, every neighbor of $v$ in $G_{2}$ has degree at least 3 in $G$ in this case. If $G_{2}-v$ is connected, then removing from $G$ an arbitrary edge joining $v$ with a vertex in $N_{v}$ produces a connected graph with minimum degree 2, contradicting the edge-minimality of $G$. Hence, $G_{2}-v$ is disconnected. Therefore at least one new edge incident with $w$ was added to $G_{2}-v$ to guarantee that $G_{2}^{*}$ is connected. Let $f=w x$ be such an added edge. We note that $\{w, x\} \subseteq N_{v}$.

As observed earlier, $G_{2}^{*} \in \mathcal{B} \cup \mathcal{C} \cup \mathcal{D} \cup \mathcal{D}_{b} \cup \mathcal{G}$. Suppose that $G_{2}^{*} \in \mathcal{C}$. Then the edge $f$ was the only edge added to $G_{2}-v$ when forming $G_{2}^{*}$, and so $G_{2}-v$ is a path whose ends, namely $w$ and $x$, are both adjacent to $v$ in $G_{2}$. By the edge-minimality of $G$, the vertex $v$ is adjacent to no other vertex on this path, implying that $G_{2}$ is a cycle and therefore $G$ is a dumb-bell, a contradiction. Therefore, $G_{2}^{*} \in \mathcal{B} \cup \mathcal{D} \cup \mathcal{D}_{b} \cup \mathcal{G}$.

We show next that the edge $f$ is a bad-edge in $G_{2}^{*}$. Suppose to the contrary that $f$ is a good-edge in $G_{2}^{*}$. Then the $\gamma_{t}^{d}\left(G_{2}^{*}\right)$-set, $S^{*}$, can be chosen to contain both $w$ and $x$. With this choice of $S^{*}$, let $S=S^{*} \cup\{u\}$. Then, $S$ is a DTD-set of $G$, and so $\gamma_{t}^{d}(G) \leq|S|+1=\gamma_{t}^{d}\left(G_{2}^{*}\right)+1$. By Observation 7 and since $G_{2}^{*} \in \mathcal{B} \cup \mathcal{D} \cup \mathcal{D}_{b} \cup \mathcal{G}$, we have that $\gamma_{t}^{d}\left(G_{2}^{*}\right) \leq n_{2}^{*} / 2=(n-4) / 2$, implying that $\gamma_{t}^{d}(G) \leq(n-2) / 2$, a contradiction. Therefore, the edge $f$ is a bad-edge in $G_{2}^{*}$.

By Observation 10 we have that $G_{2}^{*} \in\left\{D(4,4), D_{b}(3,4), D_{b}(3,3,1)\right\} \cup \mathcal{G} \backslash \mathcal{G}_{1}$. We show that $G_{2}^{*} \in$ $\mathcal{G} \backslash \mathcal{G}_{1}$. Suppose to the contrary that $G_{2}^{*} \in\left\{D(4,4), D_{b}(3,4), D_{b}(3,3,1)\right\}$. Then, by Observation 10 and by the edge-minimality of $G$ and the construction of $G_{2}^{*}$, the edge $f$ was the only edge added to $G_{2}-v$ when forming $G_{2}^{*}$. The graph $G$ is therefore determined and has order $n=11$, and it can be readily checked that $\gamma_{t}^{d}(G)=4<(n-1) / 2$, a contradiction. Therefore, $G_{2}^{*} \in \mathcal{G} \backslash \mathcal{G}_{1}$.

As observed earlier, the edge $f$ is a bad-edge in $G_{2}^{*}$. Let $v^{*}$ be the identified vertex of $G_{2}^{*}$. By Observation 10 (d), the edge $f$ is incident with the vertex $v^{*}$. We show that $G_{2}^{*} \in \mathcal{G}_{0}$. Suppose to the contrary that $G_{2}^{*} \notin \mathcal{G}_{0}$. Then, $G_{2}^{*} \in \mathcal{G}_{i}$ for some $i, 2 \leq i \leq 6$. Let $x^{*}$ be the neighbor of $v^{*}$ that belongs to the $X_{i}$-unit in $G_{2}^{*}$. By Observation 11 , the vertex $v^{*}$ is a good-vertex of $G_{2}^{*}$. Thus the $\gamma_{t}^{d}\left(G_{2}^{*}\right)$-set, $S^{*}$, can be chosen to contain $v^{*}$. Further, in each unit of type-1 or type-2 in $G_{2}^{*}$, we can choose $S^{*}$ to contain the neighbor of $v^{*}$ in that unit as well as a vertex at distance 2 from $v^{*}$ is that unit. If $v$ is not adjacent to $x^{*}$ in $G$, then the set $S^{*} \cup\{u\}$ is a DTD-set of $G$, implying that $\gamma_{t}^{d}(G) \leq\left|S^{*}\right|+1=\gamma_{t}^{d}\left(G_{2}^{*}\right)+1=\left(n_{2}^{*}-1\right) / 2+1=(n-3) / 2$, a contradiction. Hence, $v x^{*}$ is an edge of $G$. In this case, the set $\left(S^{*} \cup\{u, v\}\right) \backslash\left\{v^{*}\right\}$ is a DTD-set of $G$, implying that $\gamma_{t}^{d}(G) \leq\left|S^{*}\right|+1=(n-3) / 2$, a contradiction. Therefore, $G_{2}^{*} \in \mathcal{G}_{0}$.

The $\gamma_{t}^{d}\left(G_{2}^{*}\right)$-set, $S^{*}$, can be chosen to contain the neighbor of $v^{*}$ in each unit of type- 1 or type- 2 in $G_{2}^{*}$ as well as a vertex at distance 2 from $v^{*}$ in each unit. By the edge-minimality of $G$, and the way in which $G_{2}^{*}$ is constructed, if $z$ is a neighbor of $v$ in $G_{2}$, then either $z=v^{*}$ or $z$ is a neighbor of $v^{*}$ in $G_{2}^{*}$. Thus if $d_{G_{2}}(v) \geq 3$, then the set $S^{*} \cup\{u\}$ is a DTD-set of $G$, implying that $\gamma_{t}^{d}(G) \leq\left|S^{*}\right|+1=\gamma_{t}^{d}\left(G_{2}^{*}\right)+1=$ $\left(n_{2}^{*}-1\right) / 2+1=(n-3) / 2$, a contradiction. Hence, $d_{G_{2}}(v)=2$, implying that the ends of the edge $f$, namely $w$ and $x$, are the only neighbors of $v$ in $G_{2}$. As observed earlier, the edge $f$ is incident with the vertex $v^{*}$. Let $\left\{u^{*}, v^{*}\right\}=\{w, x\}$. If $u^{*}$ belongs to a type- 1 unit in $G_{2}^{*}$, then $G \in \mathcal{G}_{3}$. If $u^{*}$ belongs to a type-2 unit in $G_{2}^{*}$, then $G \in \mathcal{G}_{5}$. This completes the proof of Claim 1

By Claim 1, we may assume that $G_{1} \neq C_{3}$, for otherwise the desired result follows. Analogously, we may assume that $G_{2} \neq C_{3}$.

Claim $2 G_{1} \neq C_{7}$.

Proof of Claim 2. Suppose to the contrary that $G_{1}=C_{7}$. Then, $n=n_{2}+7$. Let $G_{1}$ be the cycle $u u_{1} u_{2} \ldots u_{6} u$. By Observation $18 \gamma_{t}^{d}\left(G_{2}\right) \leq\left(n_{2}-2\right) / 2$ or $G_{2} \in \mathcal{B} \cup \mathcal{C} \cup \mathcal{D} \cup \mathcal{D}_{b} \cup \mathcal{G}$. Let $S$ be a 
$\gamma_{t}^{d}\left(G_{2}\right)$-set. Suppose that $\gamma_{t}^{d}\left(G_{2}\right) \leq\left(n_{2}-1\right) / 2=n / 2-4$. Then the set $S \cup\left\{u_{3}, u_{4}, v\right\}$ is a DTDset of $G$, implying that $\gamma_{t}^{d}(G) \leq \gamma_{t}^{d}\left(G_{2}\right)+3=n / 2-1$, a contradiction. Hence, $\gamma_{t}^{d}\left(G_{2}\right) \geq n_{2} / 2$ and $G_{2} \in \mathcal{B} \cup \mathcal{C} \cup \mathcal{D} \cup \mathcal{D}_{b} \cup \mathcal{G}$. Since $G$ is not a dumb-bell, the graph $G_{2}$ is not a cycle. Thus by Observation 7. $\gamma_{t}^{d}\left(G_{2}\right)=n_{2} / 2$ and $G_{2} \in\left\{B_{3}, D_{b}(4,4), D_{b}(3,4,1), D_{b}(3,3,2)\right\}$. By Observation 11 . every vertex of $G_{2}$ is a good-vertex. In particular, the vertex $v$ is a good-vertex of $G_{2}$. Choosing the $\gamma_{t}^{d}\left(G_{2}\right)$-set, $S$, to contain the vertex $v$, we have that the set $S \cup\left\{u_{3}, u_{4}\right\}$ is a DTD-set of $G$, implying that $\gamma_{t}^{d}(G) \leq|S|+2=\gamma_{t}^{d}\left(G_{2}\right)+2=n_{2} / 2+2=(n-3) / 2$, a contradiction. This completes the proof of Claim 2 .

By Claim 2, we have $G_{1} \neq C_{7}$. Analogously, we have $G_{2} \neq C_{7}$. Since $G_{1} \notin\left\{C_{3}, C_{7}\right\}$, we have that $\gamma_{t}^{d}(G)=n_{1} / 2$ and $G_{1} \in\left\{C_{4}, C_{6}, C_{8}, C_{12}\right\} \cup\left\{B_{3}, D_{b}(4,4), D_{b}(3,4,1), D_{b}(3,3,2)\right\}$. If $\gamma_{t}^{d}\left(G_{2}\right) \leq$ $\left(n_{2}-2\right) / 2$, then $\gamma_{t}^{d}(G) \leq \gamma_{t}^{d}\left(G_{1}\right)+\gamma_{t}^{d}\left(G_{2}\right) \leq n_{1} / 2+\left(n_{2}-2\right) / 2=(n-2) / 2$, a contradiction. Hence, $\gamma_{t}^{d}\left(G_{2}\right) \geq\left(n_{2}-1\right) / 2$. By Observation $18, G_{2} \in \mathcal{B} \cup \mathcal{C} \cup \mathcal{D} \cup \mathcal{D}_{b} \cup \mathcal{G}$. By our earlier assumptions and observations, $G_{2} \notin\left\{C_{3}, C_{7}\right\}$, implying that $\gamma_{t}^{d}\left(G_{2}\right) \leq n_{2} / 2$.

Claim 3 If $G_{1}=C_{4}$, then $G=B_{10}$ or $G \in \mathcal{G}$.

Proof of Claim 3 . Suppose that $G_{1}=C_{4}$. Then, $n=n_{2}+4$. As observed earlier, $G_{2} \notin\left\{C_{3}, C_{7}\right\}$ and $G_{2} \in \mathcal{B} \cup \mathcal{C} \cup \mathcal{D} \cup \mathcal{D}_{b} \cup \mathcal{G}$. Since $G$ is not a dumb-bell, the graph $G_{2}$ is not a cycle. Thus, $G_{2} \notin \mathcal{C}$, and so $G_{2} \in \mathcal{B} \cup \mathcal{D} \cup \mathcal{D}_{b} \cup \mathcal{G}$. By the edge-minimality of $G$, the vertex $v$ is a special vertex in $G_{2}$. Recall that the graph $G_{2}^{v}$ is obtained from $G_{2}$ by adding a new vertex $v^{\prime}$ and adding the pendant edge $v v^{\prime}$.

Suppose $\gamma_{n t}^{d}\left(G_{2}^{v}\right) \leq \gamma_{t}^{d}\left(G_{2}\right)$. Let $S_{2}$ be a $\gamma_{n t}^{d}\left(G_{2}^{v}\right)$-set. Let $u_{1}$ be a neighbor of $u$ in $G_{1}$. Then the set $\left(S_{2} \backslash\left\{v^{\prime}\right\}\right) \cup\left\{u, u_{1}\right\}$ is a DTD-set of $G$, and so $\gamma_{t}^{d}(G) \leq\left|S_{2}\right|+1=\gamma_{n t}^{d}\left(G_{2}^{v}\right)+1 \leq \gamma_{t}^{d}\left(G_{2}\right)+1 \leq$ $n_{2} / 2+1=(n-2) / 2$, a contradiction. Hence, $\gamma_{n t}^{d}\left(G_{2}^{v}\right)>\gamma_{t}^{d}\left(G_{2}\right)$. Applying Observation 14 to the graph $G_{2}$ and the special vertex $v$ of $G_{2}$, one of the three conditions (b), (c) or (d) in the statement of the observation hold. We consider each of the three conditions in turn.

If $G_{2}=B_{1}$ and $v$ has degree 3 in $G_{2}$, then $G=B_{10}$. If $G_{2} \in \mathcal{G}$ and $v$ is the identified vertex of $G_{2}$, then $G \in \mathcal{G}$ and $G$ has one additional type-2 unit than does $G_{2}$. Suppose $G_{2} \in \mathcal{G}_{0}$ and $v$ is a neighbor of the identified vertex of $G_{2}$. Then, $G_{2}=G_{0}(i, j)$ for some $i, j$ where $i+j \geq 2$ and either $v$ belongs to a type- 1 unit or a type- 2 unit of $G_{2}$. If $v$ belongs to a type- 1 unit of $G_{2}$, then $G \in G_{5}(i-1, j) \in \mathcal{G}_{5}$. If $v$ belongs to a type- 2 unit of $G_{2}$, then $G \in G_{6}(i, j-1) \in \mathcal{G}_{6}$. Hence if $G_{2} \in \mathcal{G}_{0}$, then $G \in \mathcal{G}$. This completes the proof of Claim 3 .

By Claim 3, we may assume that $G_{1} \neq C_{4}$, for otherwise the desired result follows. Analogously, we may assume that $G_{2} \neq C_{4}$. By the edge-minimality of $G$, the vertex $u$ is a special vertex in $G_{1}$ and the vertex $v$ is a special vertex in $G_{2}$. Recall that the graph $G_{1}^{u}$ is obtained from $G_{1}$ by adding a new vertex $u^{\prime}$ and adding the pendant edge $u u^{\prime}$. By our earlier observations, $G_{1} \in\left\{C_{6}, C_{8}, C_{12}\right\} \cup$ $\left\{B_{3}, D_{b}(4,4), D_{b}(3,4,1), D_{b}(3,3,2)\right\}$. Further, $G_{2} \notin\left\{C_{3}, C_{4}, C_{7}\right\}$ and $G_{2} \in \mathcal{B} \cup \mathcal{C} \cup \mathcal{D} \cup \mathcal{D}_{b} \cup \mathcal{G}$.

Claim $4 G_{2} \in \mathcal{G}_{0}$ and that $v$ is the identified vertex of $G_{2}$.

Proof of Claim 4. Suppose that $v$ is a good-vertex of $G_{2}$. Let $S_{2}$ be a $\gamma_{t}^{d}\left(G_{2}\right)$-set that contains the vertex $v$. By Observation 14, we have that $\gamma_{n t}^{d}\left(G_{1}^{u}\right) \leq \gamma_{t}^{d}\left(G_{1}\right)$. Let $S_{1}$ be a $\gamma_{n t}^{d}\left(G_{1}^{u}\right)$-set and note that $u^{\prime} \in S_{1}$. Then the set $\left(S_{1} \backslash\left\{u^{\prime}\right\}\right) \cup S_{2}$ is a DTD-set of $G$, and so $\gamma_{t}^{d}(G) \leq\left|S_{1}\right|+\left|S_{2}\right|-1=\gamma_{n t}^{d}\left(G_{1}^{u}\right)+\gamma_{t}^{d}\left(G_{2}\right)-1 \leq$ $\gamma_{t}^{d}\left(G_{1}\right)+\gamma_{t}^{d}\left(G_{2}\right)-1 \leq n_{1} / 2+n_{2} / 2-1=(n-2) / 2$, a contradiction. Hence, $v$ is a bad-vertex of $G_{2}$. Applying Observation 11 to the graph $G_{2}$, we have that $G_{2} \in\left\{D(4,4), D_{b}(3,4), D_{b}(3,3,1)\right\}$ or $G_{2} \in \mathcal{G}_{0}$. Further, if $G_{2} \in \mathcal{G}_{0}$, then $v$ is the identified vertex of $G_{2}$. 
By Observation 11 every vertex of $G_{1}$ is a good-vertex of $G_{1}$. Let $D_{1}$ be a $\gamma_{t}^{d}\left(G_{1}\right)$-set that contains the vertex $u$. Suppose that $\gamma_{n t}^{d}\left(G_{2}^{v}\right) \leq \gamma_{t}^{d}\left(G_{2}\right)$. Let $D_{2}$ be a $\gamma_{n t}^{d}\left(G_{2}^{v}\right)$-set. Then the set $D_{1} \cup\left(D_{2} \backslash\left\{v^{\prime}\right\}\right)$ is a DTD-set of $G$, and so $\gamma_{t}^{d}(G) \leq\left|D_{1}\right|+\left|D_{2}\right|-1 \leq \gamma_{t}^{d}\left(G_{1}\right)+\gamma_{n t}^{d}\left(G_{2}^{v}\right)-1 \leq \gamma_{t}^{d}\left(G_{1}\right)+\gamma_{t}^{d}\left(G_{2}\right)-1 \leq$ $n_{1} / 2+n_{2} / 2-1=(n-2) / 2$, a contradiction. Hence, $\gamma_{n t}^{d}\left(G_{2}^{v}\right)>\gamma_{t}^{d}\left(G_{2}\right)$. By Observation 14 . we therefore have that $G_{2} \notin\left\{D(4,4), D_{b}(3,4), D_{b}(3,3,1)\right\}$, implying that $G_{2} \in \mathcal{G}_{0}$ and that $v$ is the identified vertex of $G_{2}$. This completes the proof of Claim 4 .

We now return to the proof of Lemma 19 one last time. By Claim 4, $G_{2} \in \mathcal{G}_{0}$ and $v$ is the identified vertex of $G_{2}$. Let $S_{1}$ be a $\gamma_{n t}^{d}\left(G_{1}^{u}\right)$-set and note that $u^{\prime} \in S_{1}$. Let $S_{2}$ be a $\gamma_{t}^{d}\left(G_{2}\right)$-set that contains all neighbors of $v$ in $G_{2}$ and a vertex at distance 2 from $v$ in each unit of $G_{2}$. Then, $\gamma_{t}^{d}\left(G_{2}\right)=\left|S_{2}\right|=$ $\left(n_{2}-1\right) / 2$. As observed earlier, we have that $G_{1} \in\left\{C_{6}, C_{8}, C_{12}, B_{3}, D_{b}(4,4), D_{b}(3,4,1), D_{b}(3,3,2)\right\}$. We consider the seven possibilities in turn.

If $G=C_{6}$, then $G \in \mathcal{G}_{2}$.

If $G_{1}=C_{8}$, let $G_{1}$ be given by $u u_{1} u_{2} \ldots u_{7} u$. Then the set $S_{2} \cup\left\{u, u_{3}, u_{4}\right\}$ is a DTD-set of $G$, and so $\gamma_{t}^{d}(G) \leq\left|S_{2}\right|+3=\gamma_{t}^{d}\left(G_{1}\right)+\gamma_{t}^{d}\left(G_{2}\right)-1=n_{1} / 2+\left(n_{2}-1\right) / 2-1=(n-3) / 2$, a contradiction. Hence, $G_{1} \neq C_{8}$.

Suppose $G_{1} \in\left\{C_{12}, B_{3}\right\}$. Then, $\gamma_{n t}^{d}\left(G_{1}^{u}\right)=\gamma_{t}^{d}\left(G_{1}\right)-1$. The set $\left(S_{1} \backslash\left\{u^{\prime}\right\}\right) \cup\left(S_{2} \cup\{v\}\right)$ is a DTD-set of $G$, and so $\gamma_{t}^{d}(G) \leq\left|S_{1}\right|+\left|S_{2}\right|=\gamma_{t}^{d}\left(G_{1}\right)+\gamma_{t}^{d}\left(G_{2}\right)-1=n_{1} / 2+\left(n_{2}-1\right) / 2-1=(n-3) / 2$, a contradiction. Hence, $G_{1} \notin\left\{C_{12}, B_{3}\right\}$.

Suppose $G_{1}=D_{b}(4,4)$. If $u$ has degree 2 in $G_{1}$, then $\gamma_{n t}^{d}\left(G_{1}^{u}\right)=\gamma_{t}^{d}\left(G_{1}\right)-1$, and as before we obtain a contradiction. Hence, $d_{G_{1}}(u)=3$. But then $G \in \mathcal{G}_{6}$.

Suppose $G_{1}=D_{b}(3,4,1)$. Then, $G_{1}$ can be obtained from a path $u_{1} u_{2} \ldots u_{8}$ by adding the edges $u_{1} u_{3}$ and $u_{5} u_{8}$. Since $u$ is a special vertex of $G_{1}$, we note that $u \in\left\{u_{3}, u_{4}, u_{5}, u_{7}\right\}$. If $u=u_{3}$, let $S=S_{2} \cup\left\{u_{3}, u_{5}, u_{6}\right\}$. If $u=u_{7}$, let $S=S_{2} \cup\left\{u_{3}, u_{4}, u_{5}\right\}$. In both cases, $S$ is a DTD-set of $G$, and so $\gamma_{t}^{d}(G) \leq|S|=\left|S_{2}\right|+3=\gamma_{t}^{d}\left(G_{1}\right)+\gamma_{t}^{d}\left(G_{2}\right)-1=(n-3) / 2$, a contradiction. Hence, $u \in\left\{u_{4}, u_{5}\right\}$. If $u=u_{4}$, then $G \in \mathcal{G}_{5}$. If $u=u_{5}$, then $G \in \mathcal{G}_{4}$.

Suppose $G_{1}=D_{b}(3,3,2)$. Then, $G_{1}$ can be obtained from a path $u_{1} u_{2} \ldots u_{8}$ by adding the edges $u_{1} u_{3}$ and $u_{6} u_{8}$. Since $u$ is a special vertex of $G_{1}$, we note that $u \in\left\{u_{3}, u_{4}, u_{5}, u_{6}\right\}$. By symmetry, we may assume that $u \in\left\{u_{3}, u_{4}\right\}$. If $u=u_{3}$, then $S_{2} \cup\left\{u_{3}, u_{5}, u_{6}\right\}$ is a DTD-set of $G$, and so $\gamma_{t}^{d}(G) \leq\left|S_{2}\right|+3=\gamma_{t}^{d}\left(G_{1}\right)+\gamma_{t}^{d}\left(G_{2}\right)-1=(n-3) / 2$, a contradiction. Hence, $u=u_{4}$. But then $G \in \mathcal{G}_{3}$.

Hence we have shown that $G_{1} \in\left\{C_{6}, D_{b}(4,4), D_{b}(3,4,1), D_{b}(3,3,2)\right\}$ and that $G \in \mathcal{G}$. This completes the proof of Lemma 19.

By Lemma 19 , we may assume that $\mathcal{L}$ is not an independent set, for otherwise the desired result follows.

Lemma 20 If $G$ contains a path on six vertices each internal vertex of which has degree 2 in $G$ and whose end vertices are not adjacent, then $G \in\left\{B_{3}, B_{4}, B_{5}, B_{7}, B_{11}\right\}$.

Proof of Lemma 20, Let $u$ and $v$ be non-adjacent vertices in $G$ joined by a path $u w_{1} w_{2} w_{3} w_{4} v$ every internal vertex of which has degree 2 in $G$. Let $G^{\prime}$ be the graph obtained from $G$ by removing the vertices $w_{1}, w_{2}, w_{3}$ and $w_{4}$, and adding the edge $u v$. Then, $G^{\prime}$ is a connected graph of order $n^{\prime}=n-4$ with $\delta\left(G^{\prime}\right) \geq 2$. By Observation 6 , $\gamma_{t}^{d}(G) \leq \gamma_{t}^{d}\left(G^{\prime}\right)+2$. It follows that if $\gamma_{t}^{d}\left(G^{\prime}\right) \leq\left(n^{\prime}-2\right) / 2$, then $\gamma_{t}^{d}(G) \leq(n-6) / 2+2=(n-2) / 2$, a contradiction. Hence, $\gamma_{t}^{d}\left(G^{\prime}\right) \geq\left(n^{\prime}-1\right) / 2$. Let $F=G^{\prime}-e$. Then, $F$ has order $n^{\prime}$ and either $F$ is disconnected or $\delta(F)=1$ or $F$ is connected and $\delta(F) \geq 2$.

Claim 5 If $F$ is disconnected or $\delta(F)=1$, then $G \in\left\{B_{5}, B_{11}\right\} \subset \mathcal{B}$. 
Proof of Claim 5. Assume that $F$ is disconnected or $\delta(F)=1$. Then, $G^{\prime}$ is an edge-minimal graph. As observed earlier, $\gamma_{t}^{d}\left(G^{\prime}\right) \geq\left(n^{\prime}-1\right) / 2$. Thus, $G^{\prime}$ is a $\frac{1}{2}$-minimal graph. Applying the induction hypothesis to the graph $G^{\prime}$, we see that $G^{\prime} \in \mathcal{B} \cup \mathcal{C} \cup \mathcal{D} \cup \mathcal{D}_{b} \cup \mathcal{G}$. If $G^{\prime} \in \mathcal{C} \cup \mathcal{D} \cup \mathcal{D}_{b} \cup \mathcal{G}_{b}$, then $G \in \mathcal{C} \cup \mathcal{D} \cup \mathcal{D}_{b} \cup \mathcal{G}_{b}$, contradicting our previous assumptions. Hence, $G^{\prime} \in \mathcal{B} \cup\left(\mathcal{G} \backslash \mathcal{G}_{b}\right)$.

We show that $G^{\prime} \in \mathcal{B}$. Suppose to the contrary that $G^{\prime} \notin \mathcal{B}$. Then, $G^{\prime} \in \mathcal{G} \backslash \mathcal{G}_{b}$. Let $x$ be the identified vertex of $G$. Suppose that $G^{\prime} \in \mathcal{G}_{0}$, and so $G^{\prime}=G_{0}\left(n_{1}, n_{2}\right)$ for some $n_{1} \geq 0$ and $n_{2} \geq 0$ where $n_{1}+n_{2} \geq 2$. Since $G^{\prime} \notin \mathcal{G}_{b}$, we note that $n_{1}+n_{2} \geq 3$. By Observation 12 , there is a $\gamma_{t}^{d}\left(G^{\prime}\right)$-set, $S^{\prime}$, such that $v \notin S^{\prime}, N(v) \subset S^{\prime}$ and $S^{\prime}$ totally dominates $N(v)$ in $G^{\prime}$. In particular, we note that in each unit in $G^{\prime}$ there is exactly one vertex at distance 2 from $x$ in $G^{\prime}$ that belongs to $S^{\prime}$. If $e$ is a bridge of $G^{\prime}$, then we may assume, renaming vertices if necessary, that $x$ is incident with $e$ and that $x=u$. In this case, we let $S=S^{\prime} \cup\left\{w_{1}\right\}$. If $e$ belongs to a 3 -cycle in $G^{\prime}$, then renaming vertices, if necessary, we may assume that this 3-cycle is given by $z u v z$ and that $x y z$ is a path in $G^{\prime}$. In this case, we note that $\{y, z\} \subset S^{\prime}$ and we let $S=(S \backslash\{z\}) \cup\left\{w_{2}, w_{3}\right\}$. If $e$ belongs to a 4-cycle in $G^{\prime}$, then renaming vertices, if necessary, we may assume that this 4-cycle is given by yuvzy and that $x y$ is a path in $G^{\prime}$. In this case, we may assume that $\{y, z\} \subset S^{\prime}$ (since if $u \in S^{\prime}$, we simply replace $u$ in $S^{\prime}$ by $z$ ) and let $S=(S \backslash\{z\}) \cup\left\{w_{2}, w_{3}\right\}$. In all three cases, the set $S$ is a DTD-set of $G$, and so $\gamma_{t}^{d}(G) \leq|S|+1=\left(n^{\prime}-1\right) / 2+1=(n-3) / 2$, a contradiction. Hence, $G^{\prime} \notin \mathcal{G}_{0}$.

Suppose that $G^{\prime} \in \mathcal{G}_{1}$, and so $G^{\prime}=G_{1}\left(n_{1}, n_{2}\right)$ for some $n_{1} \geq 0$ and $n_{2} \geq 0$ where $n_{1}+n_{2} \geq 1$. Since $G^{\prime} \notin \mathcal{G}_{b}$, we note that $n_{1}+n_{2} \geq 2$. By Observation 12 , there is a $\gamma_{t}^{d}\left(G^{\prime}\right)$-set, $S^{\prime}$, such that $v \notin S^{\prime}$ and $N(v) \subset S^{\prime}$. Further we can choose such a set $S^{\prime}$ so that each unit in $G^{\prime}$ contains a vertex at distance 2 from $x$ in $G^{\prime}$ that belongs to $S^{\prime}$. If $n_{2} \geq 1$, then since $\mathcal{L}$ is an independent set, we have that $n_{2}=1$ and that $e$ is the edge that joins $x$ to the vertex of the 4-cycle in $G^{\prime}$. Renaming $u$ and $v$, if necessary, we may assume that $u=x$. In this case, we let $S=S^{\prime} \cup\left\{w_{1}\right\}$. Then, $S$ is a DTD-set of $G$, and so $\gamma_{t}^{d}(G) \leq|S|+1=\left(n^{\prime}-1\right) / 2+1=(n-3) / 2$, a contradiction. Hence, $G^{\prime}=G_{1}\left(n_{1}, 0\right)$ for some $n_{1} \geq 2$. If $e$ belongs to the 5 -cycle in $G^{\prime}$, then we may assume, renaming vertices if necessary, that $x$ is incident with $e$ and that $x=u$. In this case, we note that $v \in S^{\prime}$ and we let $S=\left(S^{\prime} \backslash\{v\}\right) \cup\left\{w_{3}, w_{4}\right\}$. If $e$ is a bridge of $G$, then we may assume, renaming vertices if necessary, that $x$ is incident with $e$ and that $x=u$. In this case, we let $S=S^{\prime} \cup\left\{w_{1}\right\}$. If $e$ belongs to a 3-cycle in $G^{\prime}$, then renaming vertices, if necessary, we may assume that this 3 -cycle is given by zuvz and that $x y z$ is a path in $G^{\prime}$. In this case, we note that $\{y, z\} \subset S^{\prime}$ and we let $S=(S \backslash\{z\}) \cup\left\{w_{2}, w_{3}\right\}$. In all three cases, the set $S$ is a DTD-set of $G$, and so $\gamma_{t}^{d}(G) \leq|S|+1=\left(n^{\prime}-1\right) / 2+1=(n-3) / 2$, a contradiction. Hence, $G^{\prime} \notin \mathcal{G}_{1}$.

Suppose that $G^{\prime} \in \mathcal{G}_{2}$, and so $G^{\prime}=G_{2}\left(n_{1}, n_{2}\right)$ for some $n_{1} \geq 0$ and $n_{2} \geq 0$ where $n_{1}+n_{2} \geq 1$. Since $G^{\prime} \notin \mathcal{G}_{b}$, we note that $n_{1}+n_{2} \geq 2$. By Observation 12 , there is a $\gamma_{t}^{d}\left(G^{\prime}\right)$-set, $S^{\prime}$, such that $v \notin S^{\prime}$, $N(v) \subset S^{\prime}$ and $S^{\prime}$ totally dominates $N(v)$ in $G^{\prime}$. Since $\mathcal{L}$ is an independent set, $e$ is the edge that joins $x$ to the vertex of the 6 -cycle in $G^{\prime}$. Renaming $u$ and $v$, if necessary, we may assume that $u=x$. Let $C: v v_{1} v_{2} v_{3} v_{4} v_{5} v$ be the 6 -cycle in $G^{\prime}$. We may choose $S^{\prime}$ so that $S \cap V(C)=\left\{v, v_{1}, v_{5}\right\}$. With this choice of the set $S^{\prime}$, the set $S^{\prime} \cup\left\{w_{4}\right\}$ is a DTD-set of $G$, and so $\gamma_{t}^{d}(G) \leq\left|S^{\prime}\right|+1=\left(n^{\prime}-1\right) / 2+1=(n-3) / 2$, a contradiction. Hence, $G^{\prime} \notin \mathcal{G}_{2}$.

Suppose that $G^{\prime} \in \mathcal{G}_{3}$. Since $\mathcal{L}$ is an independent set in $G$, either $G^{\prime}=G_{3}(1,0)$ or $G^{\prime}=G_{3}(0,1)$. In both cases, $e$ is the edge joining the two vertices of degree 3 in $G^{\prime}$. The graph $G$ is therefore determined and has order $n=17$ and $\gamma_{t}^{d}(G) \leq 7=(n-3) / 2$, a contradiction. Hence, $G^{\prime} \notin \mathcal{G}_{3}$.

Suppose that $G^{\prime} \in \mathcal{G}_{4}$. Since $\mathcal{L}$ is an independent set in $G$, either $G^{\prime}=G_{4}(1,0)$ or $G^{\prime}=G_{4}(0,1)$. In both cases, $G^{\prime}$ has order $n^{\prime}=13$ and, up to isomorphism, there are five different choice for the edge $e$. This gives rise to a total of ten possible (non-isomorphic) constructions for the graph $G$. However in all 
ten cases, we have $n=17$ and $\gamma_{t}^{d}(G) \leq 7=(n-3) / 2$, a contradiction. Hence, $G^{\prime} \notin \mathcal{G}_{4}$.

If $G^{\prime} \in \mathcal{G}_{5}$, then for every possible choice of the edge $e$, we will always produce two adjacent large vertices in $G$, contradicting our assumption that $\mathcal{L}$ is an independent set. Hence, $G^{\prime} \notin \mathcal{G}_{5}$.

Suppose that $G^{\prime} \in \mathcal{G}_{6}$. Since $\mathcal{L}$ is an independent set in $G$, either $G^{\prime}=G_{6}(1,0)$ or $G^{\prime}=G_{6}(0,1)$. In both cases, $e$ is the edge joining the two large vertices in $G^{\prime}$. The graph $G$ is therefore determined and has order $n=17$ and $\gamma_{t}^{d}(G) \leq 7=(n-3) / 2$, a contradiction. Hence, $G^{\prime} \notin \mathcal{G}_{6}$. Therefore, $G^{\prime} \in \mathcal{B}$.

We show next that $G^{\prime} \in\left\{B_{1}, B_{5}\right\}$. As observed earlier, $G^{\prime} \in \mathcal{B}$. We wish to show that $G^{\prime} \in\left\{B_{1}, B_{5}\right\}$. Suppose to the contrary that $G^{\prime} \notin\left\{B_{1}, B_{5}\right\}$. Suppose that $G^{\prime}=B_{2}$. Up to isomorphism, there are two different choices for the edge $e$. This gives rise to two possible (non-isomorphic) constructions for the graph $G$ from the graph $G^{\prime}$. However in both cases, we have $n=11$ and $\gamma_{t}^{d}(G) \leq 4=(n-3) / 2$, a contradiction.

Suppose that $G^{\prime}=B_{3}$. Up to isomorphism, there are two different choices for the edge $e$. This gives rise to two possible (non-isomorphic) constructions for the graph $G$ from the graph $G^{\prime}$. However in both cases, we have $n=12$ and $\gamma_{t}^{d}(G) \leq 5=(n-2) / 2$, a contradiction.

Suppose that $G^{\prime} \in\left\{B_{4}, B_{6}, B_{7}, B_{8}, B_{9}, B_{10}\right\}$. Then, $n^{\prime}=9$, and so $n=13$. If $G^{\prime} \in\left\{B_{4}, B_{6}, B_{8}\right\}$, then up to isomorphism, there are two different choices for the edge $e$. If $G^{\prime} \in\left\{B_{7}, B_{9}, B_{10}\right\}$, then up to isomorphism, there are three different choices for the edge $e$. In all cases, the resulting graph $G$ constructed from $G^{\prime}$ satisfies $\gamma_{t}^{d}(G) \leq 5=(n-3) / 2$, a contradiction.

Suppose that $G^{\prime}=B_{11}$. Up to isomorphism, there are two different choices for the edge $e$. This gives rise to two possible (non-isomorphic) constructions for the graph $G$ from the graph $G^{\prime}$. However in both cases, we have $n=17$ and $\gamma_{t}^{d}(G) \leq 7=(n-3) / 2$, a contradiction. Since all the above cases produce a contradiction, we deduce that $G^{\prime} \in\left\{B_{1}, B_{5}\right\}$.

If $G^{\prime}=B_{1}$, then $G=B_{5}$. Suppose $G^{\prime}=B_{5}$. Then, $n^{\prime}=9$ and $n=13$. Up to isomorphism, there are two different choices for the edge $e$. If $e$ is on one of the two paths of length 2 that join the two vertices of degree 3 in $G^{\prime}$, then $\gamma_{t}^{d}(G) \leq 5=(n-3) / 2$, a contradiction. Hence, $e$ is on the path of length 6 that join the two vertices of degree 3 in $G^{\prime}$. In this case, $G=B_{11}$. This completes the proof of Claim 5 .

We next consider the case when $F$ is connected and $\delta(F) \geq 2$.

Claim 6 If $F$ is connected and $\delta(F) \geq 2$, then $G \in\left\{B_{3}, B_{4}, B_{7}\right\}$.

Proof of Claim 6. Assume that $F$ is connected and $\delta(F) \geq 2$. By the edge-minimality of $G$ and since $\gamma_{t}^{d}(F) \geq \gamma_{t}^{d}\left(G^{\prime}\right) \geq\left(n^{\prime}-1\right) / 2$, the graph $F$ is a $\frac{1}{2}$-minimal graph. By the inductive hypothesis, $F \in \mathcal{B} \cup \mathcal{C} \cup \mathcal{D} \cup \mathcal{D}_{b} \cup \mathcal{G}$.

We show that both $u$ and $v$ are special vertices in $F$. Suppose to the contrary that $u$ is not a special vertex in $F$. Then, there is an edge $f$ in $F$ which may be removed without disconnecting $F$ and with no vertex, except possibly for the vertex $u$, having degree 1 in $F$. But then $\delta(G-f) \geq 2$ and $G$ is connected, contradicting the edge-minimality of $G$. Hence, $u$ is a special vertex in $F$. Analogously, $v$ is a special vertex in $F$. Therefore, $u$ and $v$ are special vertices in $F$.

Recall from Section 3.2, that for a graph $H$ and vertex $x$ in $H$, the graph obtained from $H$ by adding a new vertex $x^{\prime}$ and adding the pendant edge $x x^{\prime}$ is denoted by $H^{x}$.

Let $x \in\{u, v\}$ and suppose, to the contrary, that $\gamma_{n t}^{d}\left(F^{x}\right) \leq \gamma_{t}^{d}(F)=\gamma_{t}^{d}\left(G^{\prime}-e\right)$ but there exists a $\gamma_{n t}^{d}\left(F^{x}\right)$-set, $S$ say, which DT-dominates $x^{\prime}$. Renaming $u$ and $v$ if necessary, we may assume that $x=v$. Then the set $\left(S \backslash\left\{v^{\prime}\right\}\right) \cup\left\{u, w_{4}\right\}$ is a DTD-set of $G$, and so $\gamma_{t}^{d}(G) \leq|S|+1=\gamma_{t}^{d}(F)+1$. If $\gamma_{t}^{d}(F) \leq n^{\prime} / 2$, then $\gamma_{t}^{d}(G) \leq n^{\prime} / 2+1=(n-4) / 2+2=(n-2) / 2$, a contradiction. Hence, 
$\gamma_{t}^{d}(F)=\left(n^{\prime}+1\right) / 2$. By Observation $7, F \in\left\{C_{3}, C_{7}\right\}$. Since $u$ and $v$ are not adjacent in $F$, the case $F=C_{3}$ cannot occur. Therefore, $F=C_{7}$ and $u$ and $v$ are at distance either 2 or 3 apart in $F$. This gives rise to two possible constructions for the graph $G$ from the graph $F$. However in both cases, we have $n=11$ and $\gamma_{t}^{d}(G) \leq 4=(n-3) / 2$, a contradiction. Therefore, if $x \in\{u, v\}$ and $\gamma_{n t}^{d}\left(F^{x}\right) \leq \gamma_{t}^{d}(F)$, then there is no $\gamma_{n t}^{d}\left(F^{x}\right)$-set which DT-dominates $x^{\prime}$.

Let $x \in\{u, v\}$ and suppose, to the contrary, that $\gamma_{n t}^{d}\left(F^{x}\right) \leq \gamma_{t}^{d}(F)$. Renaming $u$ and $v$ if necessary, we may assume that $x=v$. Thus, there is no $\gamma_{n t}^{d}\left(F^{v}\right)$-set which DT-dominates $v^{\prime}$. By Observation 15 , either $F=D(4,4)$ and $v$ is at distance 2 from the vertex of degree 4 in $F$ or $F \in\left\{D_{b}(3,4), D_{b}(3,3,1)\right\}$, $d_{F}(v)=3$, and $v$ belongs to a 3 -cycle in $F$.

Suppose that $F=D(4,4)$ and $v$ is at distance 2 from the vertex of degree 4 in $F$. Since $\mathcal{L}$ is an independent set in $G$, and since $u$ and $v$ are not adjacent in $F$, either $u$ is the vertex of degree 4 in $F$ or $u$ is the vertex at distance 2 from $v$ in $F$.

Suppose that $F=D_{b}(3,4)$. Then, $v$ is the vertex of degree 3 in $F$ that belongs to a 3-cycle. Since $\mathcal{L}$ is an independent set in $G$, and since $u$ and $v$ are not adjacent in $F$, the vertex $u$ is the vertex in $F$ at distance 3 from $v$ in $F$.

Suppose that $F=D_{b}(3,3,1)$. Then, $v$ is one of the two vertices of degree 3 in $F$ that belongs to a 3 -cycle. Since $\mathcal{L}$ is an independent set in $G$, and since $u$ and $v$ are not adjacent in $F$, the vertex $u$ is the other vertex in $F$ of degree 3 in $F$ that belongs to a 3-cycle.

In all the above cases, the graph $G$ is determined. In particular, $G$ has order $n=11$ and $\gamma_{t}^{d}(G) \leq 4 \leq$ $(n-3) / 2$, a contradiction. Therefore, if $x \in\{u, v\}$, then $\gamma_{n t}^{d}\left(F^{x}\right)>\gamma_{t}^{d}(F)$.

Since $\gamma_{n t}^{d}\left(F^{u}\right)>\gamma_{t}^{d}(F)$, Observation 14 applies to the graph $F$ and the special vertex $u$. Analogously, since $\gamma_{n t}^{d}\left(F^{v}\right)>\gamma_{t}^{d}(F)$, Observation 14 applies to the graph $F$ and the special vertex $v$. Since there is only one identified vertex in a good-graph (that belongs to the family $\mathcal{G}$ ), we therefore have that either $F \in\left\{C_{4}, C_{5}\right\}$ or $F=B_{1}$ and $u$ and $v$ are the two vertices of degree 3 in $F$. If $F=C_{4}$, then $u$ and $v$ are at distance 2 apart in $F$, implying that $G=B_{3}$. If $F=C_{5}$, then $u$ and $v$ are at distance 2 apart in $F$, implying that $G=B_{7}$. If $F=B_{1}$, then $u$ and $v$ are the two vertices of degree 3 in $F$, implying that $G=B_{4}$. Thus we have shown that $G \in\left\{B_{3}, B_{4}, B_{7}\right\}$. This completes the proof of Claim 6 .

The proof of Lemma 20 follows immediately from Lemma 5 and Lemma 6 .

By Lemma 20, we may assume that $G$ does not contain a path on six vertices each internal vertex of which has degree 2 in $G$ and whose end vertices are not adjacent, for otherwise the desired result follows.

Lemma 21 If $G$ contains a vertex of degree 3 adjacent to ends of a 2-handle, then $G=B_{9}$ or $G \in \mathcal{G}$.

Proof of Lemma 21. Assume that $G$ contains a vertex of degree 3 adjacent to ends of a 2-handle, $C$. Let $P$ be the 2-path which has an end adjacent to $u$, and let $v$ be the other large vertex adjacent with an end of $P$. Let $w$ be a neighbor of $u$ on $C$, let $z$ be the neighbor of $u$ on $P$, and let $y$ be the end of $P$ different from $z$ (possibly, $y=z$ ). Let $C$ contain $r-1$ vertices and $P$ contain $s$ vertices. By Lemma 20, $3 \leq r \leq 6$, and $1 \leq s \leq 3$. Let $G_{1}=G[V(C) \cup\{u\} \cup V(P)]$ and let $G_{2}=G-V\left(G_{1}\right)$. Then, $G_{1}$ is a key $L_{r, s}$. Further, $G_{2}$ is a connected graph with $\delta\left(G_{2}\right) \geq 2$ and $v \in V\left(G_{2}\right)$. For $i=1,2$, let $G_{i}$ have order $n_{i}$, and so $n=n_{1}+n_{2}$. We note that $4 \leq n_{1} \leq 9$. The following result is straightforward to verify.

Claim $7\left(n_{1}-2\right) / 2 \leq \gamma_{t}^{d}\left(G_{1}\right) \leq\left(n_{1}+2\right) / 2$. More precisely, the following holds.

- $\gamma_{t}^{d}\left(G_{1}\right)=\left(n_{1}+1\right) / 2$ if and only if $G_{1}=L_{4,3}$.

- $\gamma_{t}^{d}\left(G_{1}\right)=n_{1} / 2$ if and only if $G_{1} \in\left\{L_{3,1}, L_{3,3}, L_{4,2}, L_{5,3}\right\}$. 
- $\gamma_{t}^{d}\left(G_{1}\right)=\left(n_{1}-1\right) / 2$ if and only if $G_{1} \in\left\{L_{3,2}, L_{4,1}, L_{5,2}, L_{6,1}, L_{6,3}\right\}$.

- $\gamma_{t}^{d}\left(G_{1}\right)=\left(n_{1}-2\right) / 2$ if and only if $G_{1} \in\left\{L_{5,1}, L_{6,2}\right\}$.

If $G_{2}$ is a cycle, then $G$ is a dumb-bell, a contradiction. Hence, $G_{2}$ is not a cycle. In particular, $G_{2} \notin \mathcal{C}$. Since $G$ is an edge-minimal graph, so too is $G_{2}$. By Observation $18, G_{2} \in \mathcal{B} \cup \mathcal{D} \cup \mathcal{D}_{b} \cup \mathcal{G}$ or $\gamma_{t}^{d}\left(G_{2}\right) \leq\left(n_{2}-2\right) / 2$.

Claim 8 The following holds.

(a) $\left(n_{1}-1\right) / 2 \leq \gamma_{t}^{d}\left(G_{1}\right) \leq n_{1} / 2$.

(b) $\left(n_{2}-1\right) / 2 \leq \gamma_{t}^{d}\left(G_{2}\right) \leq n_{2} / 2$.

(c) $\gamma_{t}^{d}\left(G_{1}\right)=n_{1} / 2$ or $\gamma_{t}^{d}\left(G_{2}\right)=n_{2} / 2$.

Proof of Claim 8. (a) Since $\gamma_{t}^{d}(G) \leq \gamma_{t}^{d}\left(G_{1}\right)+\gamma_{t}^{d}\left(G_{2}\right)$, if $\gamma_{t}^{d}\left(G_{1}\right) \leq\left(n_{1}-2\right) / 2$, then $\gamma_{t}^{d}(G) \leq$ $\left(n_{1}-2\right) / 2+n_{2} / 2=(n-2) / 2$, a contradiction. Hence, $\gamma_{t}^{d}\left(G_{1}\right) \geq\left(n_{1}-1\right) / 2$. It remains for us to show that $\gamma_{t}^{d}\left(G_{1}\right) \leq n_{1} / 2$. Suppose to the contrary that $\gamma_{t}^{d}\left(G_{1}\right)>n_{1} / 2$. Then, by Claim $7 . G_{1}=L_{4,3}$ and $\gamma_{t}^{d}\left(G_{1}\right)=3$. Further, $G$ has order $n=n_{7}+7$. If $\gamma_{t}^{d}\left(G_{2}\right) \leq\left(n_{2}-1\right) / 2$, then $\gamma_{t}^{d}(G) \leq \gamma_{t}^{d}\left(G_{1}\right)+\gamma_{t}^{d}\left(G_{2}\right) \leq$ $3+(n-8) / 2=(n-2) / 2$, a contradiction. Therefore, $\gamma_{t}^{d}\left(G_{2}\right) \geq n_{2} / 2$. As observed earlier, $\gamma_{t}^{d}\left(G_{2}\right) \leq$ $n_{2} / 2$. Consequently, $\gamma_{t}^{d}\left(G_{2}\right)=n_{2} / 2$. By Observation 7. $G_{2} \in\left\{B_{3}, D_{b}(4,4), D_{b}(3,4,1), D_{b}(3,3,2)\right\}$. This implies by Observation 11 that every vertex of $G_{2}$ is a good-vertex. Let $S$ be a $\gamma_{t}^{d}\left(G_{2}\right)$-set that contains the vertex $v$. Let $w$ be a neighbor of $u$ on $C$. Then, $S \cup\{u, w\}$ is a DTD-set of $G$, and so $\gamma_{t}^{d}(G) \leq|S|+2=\gamma_{t}^{d}\left(G_{2}\right)+2=n_{2} / 2+2=(n-3) / 2$, a contradiction. This establishes Part (a).

(b) If $\gamma_{t}^{d}\left(G_{2}\right) \leq\left(n_{2}-2\right) / 2$, then $\gamma_{t}^{d}(G) \leq \gamma_{t}^{d}\left(G_{1}\right)+\gamma_{t}^{d}\left(G_{2}\right) \leq n_{1} / 2+\left(n_{2}-2\right) / 2=(n-2) / 2$, a contradiction. Hence, $G_{2} \in \mathcal{B} \cup \mathcal{D} \cup \mathcal{D}_{b} \cup \mathcal{G}$. Thus, by Observation $7 . \gamma_{t}^{d}\left(G_{2}\right) \leq n_{2} / 2$.

(c) Part (c) follows from Parts (a) and (b) above and the observation that $(n-1) / 2=\left(n_{1}+n_{2}-1\right) / 2 \leq$ $\gamma_{t}^{d}(G) \leq \gamma_{t}^{d}\left(G_{1}\right)+\gamma_{t}^{d}\left(G_{2}\right)$. This completes the proof of Claim 8 .

Claim 9 If $\gamma_{t}^{d}\left(G_{1}\right)=n_{1} / 2$, then $G=B_{9}$ or $G \in \mathcal{G}$.

Proof of Claim 9. Suppose that $\gamma_{t}^{d}\left(G_{1}\right)=n_{1} / 2$. By Claim 7, $G_{1} \in\left\{L_{3,1}, L_{3,3}, L_{4,2}, L_{5,3}\right\}$.

We show that $G_{1}=L_{3,1}$. Suppose the contrary that $G_{1} \in\left\{L_{3,3}, L_{4,2}, L_{5,3}\right\}$. By Claim $8, \gamma_{t}^{d}\left(G_{2}\right) \leq$ $n_{2} / 2$. If $G_{1}=L_{5,3}$, then $n_{1}=8$ and every $\gamma_{t}^{d}\left(G_{2}\right)$-set can be extended to a DTD-set of $G$ by adding to it the vertices $u, v$ and $w$, and so $\gamma_{t}^{d}(G) \leq \gamma_{t}^{d}\left(G_{2}\right)+3 \leq n_{2} / 2+3=(n-2) / 2$, a contradiction. Hence, $G_{1} \in\left\{L_{3,3}, L_{4,2}\right\}$ and $n_{1}=6$.

We show firstly that $v$ is a bad-vertex in $G_{2}$. Suppose to the contrary that $v$ is a good-vertex in $G_{2}$ and let $S$ be a $\gamma_{t}^{d}\left(G_{2}\right)$-set that contains $v$. Then, $S \cup\{u, w\}$ is a DTD-set of $G$, and so $\gamma_{t}^{d}(G) \leq|S|+2=$ $\gamma_{t}^{d}\left(G_{2}\right)+2=n_{2} / 2+2 \leq(n-2) / 2$, a contradiction. Hence, $v$ is a bad-vertex in $G_{2}$. Therefore, by Observation 11. $G_{2} \in\left\{D(4,4), D_{b}(3,4), D_{b}(3,3,1)\right\} \cup \mathcal{G}_{0}$. Further, if $G_{2} \in \mathcal{G}_{0}$, then $v$ is the identified vertex of $G_{2}$, while if $G_{2} \in\left\{D(4,4), D_{b}(3,4), D_{b}(3,3,1)\right\}$, then $v$ is a vertex at distance 2 from the central vertex of $G_{2}$.

Suppose $G_{2} \in\left\{D(4,4), D_{b}(3,4), D_{b}(3,3,1)\right\}$. Then, $n_{2}=7$ and $v$ is a vertex at distance 2 from the central vertex of $G_{2}$. In this case, $n=13$ and in all cases we have $\gamma_{t}^{d}(G) \leq 5=(n-2) / 2$, irrespective of whether $G_{1}=L_{3,3}$ or $G_{1}=L_{4,2}$. This produces a contradiction. Therefore, $G_{2} \in \mathcal{G}_{0}$ and $v$ is the identified vertex of $G_{2}$. But then by Observation 12 there is a $\gamma_{t}^{d}\left(G_{2}\right)$-set, $S$, such that all neighbors of $v$ in $G_{2}$ belong to the set $S$. Such a set $S$ can be extended to a DTD-set in $G$ by adding to it at most two vertices of $G_{1}$. Hence, $\gamma_{t}^{d}(G) \leq|S|+2 \leq\left(n_{2}-1\right) / 2+2=(n-3) / 2$, a contradiction. Therefore, $G_{1}=L_{3,1}$. Thus, $n_{1}=4$ and $n=n_{2}+4$. By Claim 8 (b) and our earlier observations, $G_{2} \in \mathcal{B} \cup \mathcal{D} \cup \mathcal{D}_{b} \cup \mathcal{G}$. 
We claim next that the vertex $v$ is a special vertex of $G_{2}$ and $\gamma_{n t}^{d}\left(G_{2}^{v}\right)>\gamma_{t}^{d}\left(G_{2}\right)$. Suppose to the contrary that $v$ is not a special vertex in $G_{2}$. Then, there is an edge $f$ in $F$ which may be removed without disconnecting $F$ and with no vertex, except possibly for the vertex $v$, having degree 1 in $G_{2}$. But then $\delta(G-f) \geq 2$ and $G$ is connected, contradicting the edge-minimality of $G$. Hence, $v$ is a special vertex of $G_{2}$. We show next that $\gamma_{n t}^{d}\left(G_{2}^{v}\right)>\gamma_{t}^{d}\left(G_{2}\right)$. Suppose to the contrary that $\gamma_{n t}^{d}\left(G_{2}^{v}\right) \leq \gamma_{t}^{d}\left(G_{2}\right)$. Let $S$ be a $\gamma_{n t}^{d}\left(G_{2}^{v}\right)$-set. In particular, $v^{\prime} \in S$. The set $(S \cup\{u, z\}) \backslash\left\{v^{\prime}\right\}$ is a DTD-set in $G$, and so $\gamma_{t}^{d}(G) \leq|S|+1 \leq n_{2} / 2+1=(n-2) / 2$, a contradiction. Therefore, the vertex $v$ is a special vertex of $G_{2}$ and $\gamma_{n t}^{d}\left(G_{2}^{v}\right)>\gamma_{t}^{d}\left(G_{2}\right)$. The graph $G_{2}$ is therefore one of the graphs listed in (b), (c) or (d) in the statement of Observation 14 . We consider the three possibilities in turn.

If $G_{2}=B_{1}$ with $v$ a vertex of degree 3 in $G_{2}$, then $G=B_{9}$. If $G_{2} \in \mathcal{G}$ and $v$ is the identified vertex of $G_{2}$, then $G_{2}=G_{k}(i, j)$ for some non-negative integers $i, j, k$, where $k \leq 6, i+j \geq 2$ if $k=0$ and $i+j \geq 1$ if $k \geq 1$. In this case, $G=G_{k}(i+1, j) \in \mathcal{G}$. Finally, if $G_{2} \in \mathcal{G}_{0}$ and $v$ is a neighbor of the identified vertex of $G_{2}$, then $G_{2}=G_{0}(i, j)$ for some non-negative integers $i, j$ where $i+j \geq 2$. If $v$ belongs to a type- 1 unit in $G_{2}$, then $G=G_{3}(i-1, j) \in \mathcal{G}_{3}$. If $v$ belongs to a type-2 unit in $G_{2}$, then $G=G_{4}(i, j-1) \in \mathcal{G}_{4}$. In both cases, $G \in \mathcal{G}$. This completes the proof of Claim 9

Claim 10 If $\gamma_{t}^{d}\left(G_{1}\right)=\left(n_{1}-1\right) / 2$, then $G \in \mathcal{G}_{3} \cup \mathcal{G}_{4} \cup \mathcal{G}_{5}$.

Proof of Claim 10. Suppose that $\gamma_{t}^{d}\left(G_{1}\right)=\left(n_{1}-1\right) / 2$. By Claim 7, $G_{1} \in\left\{L_{3,2}, L_{4,1}, L_{5,2}, L_{6,1}, L_{6,3}\right\}$. Recall that $w$ is a neighbor of $u$ on $C, z$ is the neighbor of $u$ on $P$, and $y$ is the end of $P$ different from $z$ (possibly, $y=z$ ). Let $S_{1}$ be a $\gamma_{t}^{d}\left(G_{1}\right)$-set chosen so that the neighbor of $y$ on $P$ belongs to $S_{1}$ (this is possible due to the structure of $\left.G_{1}\right)$.

Since $\gamma_{t}^{d}\left(G_{1}\right)=\left(n_{1}-1\right) / 2$, by Claim 8 we have $\gamma_{t}^{d}\left(G_{2}\right)=n_{2} / 2$. Thus by Observation $7, G_{2} \in$ $\left\{B_{3}, D_{b}(4,4), D_{b}(3,4,1), D_{b}(3,3,2)\right\}$. Since the set $\mathcal{L}$ is independent in $G$, we note that $G_{2} \neq D_{b}(4,4)$ since the two adjacent vertices of degree 3 in $G_{2}$ will also be adjacent in $G$. Further, the vertex $v$ is a special vertex of $G_{2}$.

Suppose that $G_{2}=B_{3}$. Since $G$ is edge-minimal, there are only two choices for the vertex $v$, namely, $v$ is one of the two large vertex (of degree 3) in $G_{2}$ or $v$ is a small vertex (of degree 2) at distance 2 from a large vertex in $G_{2}$. In both cases, the set $S_{1}$ can be extended to a DTD-set of $G$ by adding to it three vertices from $G_{2}$, and so $\gamma_{t}^{d}(G) \leq \gamma_{t}^{d}\left(G_{1}\right)+\gamma_{t}^{d}\left(G_{2}\right)-1=\left(n_{1}+n_{2}-3\right) / 2=(n-3) / 2$, a contradiction. Hence, $G_{2} \neq B_{3}$.

Suppose that $G_{2}=D_{b}(3,4,1)$. Then, $n=n_{1}+8$. The graph $G_{2}$ can be obtained from a path $u_{1} u_{2} \ldots u_{8}$ by joining the vertices $u_{1}$ and $u_{4}$, and the vertices $u_{6}$ and $u_{8}$. Since $v$ is a special vertex of $G_{2}$, we note that $v \in\left\{u_{2}, u_{4}, u_{5}, u_{6}\right\}$. If $v=u_{2}$, let $S=S_{1} \cup\left\{u_{4}, u_{5}, u_{6}\right\}$, while if $v=u_{6}$, let $S=$ $S_{1} \cup\left\{u_{1}, u_{4}, u_{6}\right\}$. In both cases, $S$ is a DTD-set of $G$, and so $\gamma_{t}^{d}(G) \leq|S|+3=\gamma_{t}^{d}\left(G_{1}\right)+\gamma_{t}^{d}\left(G_{2}\right)-1=$ $(n-3) / 2$, a contradiction. Hence, $v=u_{4}$ or $u=u_{5}$. If $G_{1} \in\left\{L_{5,2}, L_{6,1}\right\}$, then $n=15$ and the set $\left\{u_{1}, u_{5}, u_{6}\right\}$ can be extended to a DTD-set of $G$ by adding to it three vertices from $G_{1}$, implying that $\gamma_{t}^{d}(G) \leq 6=(n-3) / 2$, a contradiction. If $G_{1}=L_{6,3}$, then $n=17$ the set $\left\{u_{1}, u_{5}, u_{6}\right\}$ can be extended to a DTD-set of $G$ by adding to it four vertices from $G_{1}$, implying that $\gamma_{t}^{d}(G) \leq 7=(n-3) / 2$, a contradiction. Hence, $G_{1}=L_{3,2}$ or $G_{1}=L_{4,1}$. If $v=u_{4}$, then $G \in \mathcal{G}_{4}$. If $v=u_{5}$, then $G \in \mathcal{G}_{5}$. Therefore, if $G_{2}=D_{b}(3,4,1)$, then $G \in \mathcal{G}_{4} \cup \mathcal{G}_{5}$.

Suppose that $G_{2}=D_{b}(3,3,2)$. Then, $n=n_{1}+8$. The graph $G_{2}$ can be obtained from a path $u_{1} u_{2} \ldots u_{8}$ by joining the vertices $u_{1}$ and $u_{3}$, and the vertices $u_{6}$ and $u_{8}$. Since $v$ is a special vertex of $G_{2}$, we note that $v \in\left\{u_{3}, u_{4}, u_{5}, u_{6}\right\}$. Renaming vertices if necessary, we may assume that $v=u_{3}$ or $v=u_{4}$. If $v=u_{3}$, then the set $S_{1} \cup\left\{u_{3}, u_{5}, u_{6}\right\}$ is a DTD-set of $G$, implying that $\gamma_{t}^{d}(G) \leq \gamma_{t}^{d}\left(G_{1}\right)+3=$ 
$\gamma_{t}^{d}\left(G_{1}\right)+\gamma_{t}^{d}\left(G_{2}\right)-1=(n-3) / 2$, a contradiction. Hence, $v=u_{4}$. If $G_{1} \in\left\{L_{5,2}, L_{6,1}\right\}$, then $n=15$ and the set $\left\{u_{3}, u_{5}, u_{6}\right\}$ can be extended to a DTD-set of $G$ by adding to it three vertices from $G_{1}$, implying that $\gamma_{t}^{d}(G) \leq 6=(n-3) / 2$, a contradiction. If $G_{1}=L_{6,3}$, then $n=17$ the set $\left\{u_{3}, u_{5}, u_{6}\right\}$ can be extended to a DTD-set of $G$ by adding to it four vertices from $G_{1}$, implying that $\gamma_{t}^{d}(G) \leq 7=(n-3) / 2$, a contradiction. Hence, $G_{1}=L_{3,2}$ or $G_{1}=L_{4,1}$. In both cases, $G \in \mathcal{G}_{3}$. Therefore, if $G_{2}=D_{b}(3,3,2)$, then $G \in \mathcal{G}_{3}$. Claim 10 now follows from our earlier observations.

We now return to the proof of Lemma 21 By Claim $8 . \gamma_{t}^{d}\left(G_{1}\right)=n_{1} / 2$ or $\gamma_{t}^{d}\left(G_{1}\right)=\left(n_{1}-1\right) / 2$. If $\gamma_{t}^{d}\left(G_{1}\right)=n_{1} / 2$, then by Claim $9=B_{9}$ or $G \in \mathcal{G}$. If $\gamma_{t}^{d}\left(G_{1}\right)=\left(n_{1}-1\right) / 2$, then by Claim 10 . $G \in \mathcal{G}_{4} \cup \mathcal{G}_{5}$. This completes the proof of Lemma21.

By Lemma 21, we may assume that if $G$ contains a vertex adjacent to ends of a 2-handle, then such a vertex has degree at least 4 in $G$, for otherwise the desired result follows. By our earlier observations, every 2-handle in $G$ has order at most 5 .

Lemma 22 Every 2-handle in $G$ has order 2 or 5.

Proof of Lemma 22. Suppose that $G$ contains a 2-handle $C$ with $|V(C)| \in\{3,4\}$. Let $C$ be the path $v_{1} v_{2} \ldots v_{t}$, where $t \in\{3,4\}$ and let $v$ be the vertex in $G$ adjacent to both ends of $C$ (the vertices $v_{1}$ and $\left.v_{t}\right)$. By our assumptions to date, $d_{G}(v) \geq 4$. Let $N_{v}=N(v) \backslash\left\{v_{1}, v_{t}\right\}$. By Lemma 19, $N_{v}$ comprises only small vertices. We note that $\left|N_{v}\right| \geq 2$.

Let $G^{\prime}=G-V(C)$. The graph $G^{\prime}$ is a connected subgraph of $G$ with $\delta\left(G^{\prime}\right) \geq 2$. Let $G^{\prime}$ have order $n^{\prime}=n-t$. The degree of every large vertex different from $v$ is unchanged in $G$ and $G^{\prime}$, and so $G^{\prime}$ contains at least one large vertex. Hence, $G^{\prime}$ is not a cycle and, by Observation $18, G^{\prime} \in \mathcal{B} \cup \mathcal{D} \cup \mathcal{D}_{b} \cup \mathcal{G}$ or $\gamma_{t}^{d}\left(G^{\prime}\right) \leq\left(n^{\prime}-2\right) / 2$. We proceed with the following claim.

Claim $11 G^{\prime} \in \mathcal{B} \cup \mathcal{D} \cup \mathcal{D}_{b} \cup \mathcal{G}$.

Proof of Claim 11. Suppose to the contrary that $\gamma_{t}^{d}\left(G^{\prime}\right) \leq\left(n^{\prime}-2\right) / 2$. Let $S$ be a $\gamma_{t}^{d}\left(G^{\prime}\right)$-set. If $t=4$, then $S \cup\left\{v, v_{1}\right\}$ is a DTD-set of $G$, and so $\gamma_{t}^{d}(G) \leq|S|+2=\gamma_{t}^{d}\left(G^{\prime}\right)+2=\left(n^{\prime}-2\right) / 2+2=(n-2) / 2$, a contradiction. Hence, $t=3$. Analogous to the proof presented in Claim 1 (for details see Henning and Naicker), we have that $N_{v}$ is an independent set in $G$ and the only common neighbor of two vertices in $N_{v}$ is the vertex $v$.

Let $G^{*}$ be the graph obtained from $G^{\prime}-v$ by selecting a vertex $w \in N_{v}$ (of degree 1 in $G^{\prime}-v$ ) and joining it to every other vertex in $N_{v}$. Let $G^{*}$ have order $n^{*}=n^{\prime}-1=n-4$. We note that since $N_{v}$ is an independent set in $G$ and the only common neighbor of two vertices in $N_{v}$ is the vertex $v$, and by the fact that $G^{\prime}$ is edge-minimal, the graph $G^{*}$ is edge-minimal. By construction $G^{*}$ has $|\mathcal{L}|-1 \geq 1$ large vertices, implying that $G^{*}$ is not a cycle. Hence, by the inductive hypothesis $G^{*} \in \mathcal{B} \cup \mathcal{D} \cup \mathcal{D}_{b} \cup \mathcal{G}$ or $\gamma_{t}^{d}\left(G^{*}\right) \leq\left(n^{*}-2\right) / 2$.

Suppose $\gamma_{t}^{d}\left(G^{*}\right) \leq\left(n^{*}-2\right) / 2$. Let $S^{*}$ be a $\gamma_{t}^{d}\left(G^{*}\right)$-set. If $\left|S^{*} \cap N_{v}\right|=1$, let $S=S^{*} \cup\left\{v, v_{1}\right\}$. If $\left|S^{*} \cap N_{v}\right| \geq 2$, let $S=S^{*} \cup\left\{v_{1}, w\right\}$. In both cases, $S$ is a NTD-set of $G$ and $|S| \leq\left|S^{*}\right|+2$, implying that $\gamma_{t}^{d}(G) \leq \gamma_{t}^{d}\left(G^{*}\right)+2=\left(n^{*}-2\right) / 2+2=(n-2) / 2$, a contradiction. Hence, $G^{*} \in \mathcal{B} \cup \mathcal{D} \cup \mathcal{D}_{b} \cup \mathcal{G}$. Since $G^{*}$ is not a cycle, Observation 7 implies that $\gamma_{t}^{d}\left(G^{*}\right) \leq n^{*} / 2$.

Let $x \in N_{v} \backslash\{w\}$ and consider the edge $e=w x$ that was added to $G^{\prime}-v$ when constructing $G^{*}$. If $e$ is a good-edge in $G^{*}$, then every $\gamma_{t}^{d}\left(G^{*}\right)$-set containing $w$ and $x$ may be extended to a DTD-set of $G$ by adding to it the vertex $v_{1}$, implying that $\gamma_{t}^{d}(G) \leq \gamma_{t}^{d}\left(G^{*}\right)+1 \leq n^{*} / 2+1=(n-2) / 2$, a contradiction. Therefore, the edge $e$ is a bad-edge of $G^{*}$. By Observation $10, G^{*} \in\left\{D(4,4), D_{b}(3,4), D_{b}(3,3,1)\right\} \cup \mathcal{G} \backslash \mathcal{G}_{1}$. 
Suppose $G^{*} \in\left\{D(4,4), D_{b}(3,4), D_{b}(3,3,1)\right\}$. If $G^{*}=D(4,4)$, then the edge $e$ satisfies Observation 10 a) and the graph $G$ is determined. In this case, $n=11$ and $\gamma_{t}^{d}(G) \leq 4=(n-3) / 2$, a contradiction. If $G^{*}=D_{b}(3,4)$, then the edge $e$ satisfies Observation 10 (b), implying that $\mathcal{L}$ is not an independent set in $G$, a contradiction. If $G^{*}=D_{b}(3,3,1)$, then the edge $e$ satisfies Observation 10(c), a contradiction. Since all three cases produce a contradiction, $G^{*} \in \mathcal{G} \backslash \mathcal{G}_{1}$.

Let $z$ be the identified vertex of $G^{*}$. By Observation 10 d), the edge $e=w x$ is incident with the identified vertex $z$. Hence either $w=z$ or $x=z$. In both cases, when we reconstruct the original graph $G$ from $G^{*}$ the component of $G-v z$ that contains the vertex $z$ contains at least one 2-handle whose ends are adjacent to a vertex of degree 3 in $G$, contradicting our earlier assumption that a vertex of $G$ adjacent to the ends of a 2-handle has degree at least 4 in $G$. This completes the proof of Claim 11 .

We now return to the proof of Lemma22 By Claim 11. $G^{\prime} \in \mathcal{B} \cup \mathcal{D} \cup \mathcal{D}_{b} \cup \mathcal{G}$. Since $G^{\prime}$ is not a cycle, Observation 7 implies that $\gamma_{t}^{d}\left(G^{\prime}\right) \leq n^{\prime} / 2$. If $v$ is a good-vertex in $G^{\prime}$, then every $\gamma_{t}^{d}\left(G^{\prime}\right)$-set can be extended to DTD-set of $G$ by adding to it the vertex $v_{1}$, implying that in this case $\gamma_{t}^{d}(G) \leq \gamma_{t}^{d}\left(G^{\prime}\right)+1 \leq$ $n^{\prime} / 2+1$.

Claim $12 t=3$.

Proof of Claim 12. Suppose to the contrary that $t=4$. Then, $n^{\prime}=n-4$. If $v$ is a good-vertex in $G^{\prime}$, then $\gamma_{t}^{d}(G) \leq n^{\prime} / 2+1=(n-2) / 2$, a contradiction. Hence, $v$ is a bad-vertex in $G$, and so, by Observation 11 $G \in\left\{D(4,4), D_{b}(3,4), D_{b}(3,3,1)\right\} \cup \mathcal{G}_{0}$. Suppose $G^{\prime} \in \mathcal{G}_{0}$. Then, $G^{\prime}=G_{0}\left(n_{1}, n_{2}\right) \in \mathcal{G}_{0}$, where $n_{1}+n_{2} \geq 2$. By Observation 11 a), the vertex $v$ is the identified vertex of $G$. We note that $G^{\prime}$ contains $n_{1}+n_{2}$ 2-handles whose ends are adjacent to a vertex of degree 3 in $G^{\prime}$. Reconstructing the original graph $G$ from $G^{\prime}$ we therefore note that $G$ contains $n_{1}+n_{2} 2$-handles whose ends are adjacent to a vertex of degree 3 in $G$, a contradiction. Hence, $G^{\prime} \in\left\{D(4,4), D_{b}(3,4), D_{b}(3,3,1)\right\}$. By Observation 11 (b), $v$ is a vertex at distance 2 from the central vertex in $G^{\prime}$. If $G^{\prime}=D_{b}(3,4)$, then $\mathcal{L}$ is not a independent set in $G$, a contradiction. Hence, $G^{\prime} \in\left\{D(4,4), D_{b}(3,3,1)\right\}$. In both cases, the graph $G$ is determined and $\gamma_{t}^{d}(G)=4=(n-3) / 2$, a contradiction. This completes the proof of Claim 12

By Claim 12, $t=3$. Thus, $n^{\prime}=n-3$. Suppose $\gamma_{t}^{d}\left(G^{\prime}\right)=n^{\prime} / 2$. By Observation 7 and by our assumption that $\mathcal{L}$ is an independent set in $G, G^{\prime} \in\left\{B_{3}, D_{b}(3,4,1), D_{b}(3,3,2)\right\}$. Since $v \in \mathcal{L}$ and $\mathcal{L}$ is an independent set in $G$, the vertex $v$ is not adjacent to a large vertex in $G^{\prime}$. If $G^{\prime}=B_{3}$, then, up to isomorphism, there are two possible graphs $G$. In both cases, $\gamma_{t}^{d}(G)=4=(n-3) / 2$, a contradiction. Hence $G^{\prime} \in\left\{D_{b}(3,4,1), D_{b}(3,3,2)\right\}$. In both cases, however we reconstruct the original graph $G$, we produce a 2 -handle whose ends are adjacent to a vertex of degree 3 in $G$, a contradiction. Hence, $\gamma_{t}^{d}(G) \leq\left(n^{\prime}-1\right) / 2$.

If $v$ is a good-vertex in $G^{\prime}$, then $\gamma_{t}^{d}(G) \leq\left(n^{\prime}-1\right) / 2+1=(n-2) / 2$, a contradiction. Hence, $v$ is a bad-vertex in $G^{\prime}$. By Observation 11, $G^{\prime} \in\left\{D(4,4), D_{b}(3,4), D_{b}(3,3,1)\right\} \cup \mathcal{G}_{0}$. If $G^{\prime} \in \mathcal{G}_{0}$, then analogously as in the proof of Claim 12 the graph $G$ would contain a 2-handle whose ends are adjacent to a vertex of degree 3 in $G$, a contradiction. If $G^{\prime}=D_{b}(3,4)$, then $\mathcal{L}$ is not a independent set in $G$, a contradiction. Hence, $G^{\prime} \in\left\{D(4,4), D_{b}(3,3,1)\right\}$. By Observation 11 b), $v$ is a vertex at distance 2 from the central vertex in $G^{\prime}$. In both cases, the graph $G$ is determined and $\gamma_{t}^{d}(G)=4=(n-2) / 2$, a contradiction. This completes the proof of Lemma22

By Lemma 22, every 2-handle in $G$ has order 2 or 5 . We show next that in fact $G$ contains no 2-handle.

Lemma 23 There is no 2-handle in $G$. 
Proof of Lemma 23. Suppose to the contrary that $G$ contains a 2-handle, $C$. Then, $|V(C)| \in\{2,5\}$. Let $C$ be the path $v_{1} v_{2} \ldots v_{t}$, where $t \in\{2,5\}$ and let $v$ be the vertex in $G$ adjacent to both ends of $C$. By our assumptions to date, $d_{G}(v) \geq 4$ and every neighbor of $v$ has degree 2 . Let $F=G-V(C)$ and let $F$ have order $n_{F}=n-t$. By construction, $F$ is a connected subgraph of $G$ with $\delta(F) \geq 2$. By Observation 18 . $F \in \mathcal{B} \cup \mathcal{D} \cup \mathcal{D}_{b} \cup \mathcal{G}$ or $\gamma_{t}^{d}(F) \leq\left(n_{F}-2\right) / 2$.

Suppose $\gamma_{t}^{d}(F) \leq\left(n_{F}-2\right) / 2$. If $t=2$, then $n_{F}=n-2$ and every $\gamma_{t}^{d}(F)$-set can be extended to a DTD-set of $G$ by adding to it the vertex $v$, implying that $\gamma_{t}^{d}(G) \leq \gamma_{t}^{d}(F)+1 \leq\left(n_{F}-2\right) / 2+1=$ $(n-2) / 2$, a contradiction. If $t=5$, then $n_{F}=n-5$ and every $\gamma_{t}^{d}(F)$-set can be extended to a DTD-set of $G$ by adding to it the vertices $v$ and $v_{1}$, implying that $\gamma_{t}^{d}(G) \leq \gamma_{t}^{d}(F)+2 \leq\left(n_{F}-2\right) / 2+2 \leq(n-3) / 2$, a contradiction. Hence, $F \in \mathcal{B} \cup \mathcal{D} \cup \mathcal{D}_{b} \cup \mathcal{G}$. Since $|\mathcal{L}| \geq 2$, the graph $F$ has at least one large vertex different from $v$, implying that $F$ is not a cycle. Hence by Observation $7 . \gamma_{t}^{d}(F) \leq n_{F} / 2$.

We show that $v$ is a bad-vertex in $F$. Suppose to the contrary that $v$ is a good-vertex in $F$. If $t=2$, then every $\gamma_{t}^{d}(F)$-set which contains $v$ is a DTD-set of $G$, implying in this case that $\gamma_{t}^{d}(G) \leq \gamma_{t}^{d}(F) \leq$ $n_{F} / 2=(n-2) / 2$, a contradiction. If $t=5$, then every $\gamma_{t}^{d}(F)$-set which contains $v$ can be extended to a DTD-set of $G$ by adding to it the vertex $v_{1}$, implying in this case that $\gamma_{t}^{d}(G) \leq \gamma_{t}^{d}(F)+1 \leq n_{F} / 2-1=$ $(n-3) / 2$, a contradiction. Therefore, $v$ is a bad-vertex in $F$.

By Observation 11, either $F \in\left\{D(4,4), D_{b}(3,4), D_{b}(3,3,1)\right\}$ and $v$ is the vertex at distance 2 from the central vertex in $F$, or $F \in \mathcal{G}_{0}$ and $v$ is the identified vertex of $F$. In all cases, the graph $G$ is determined. If $F \in\left\{D_{b}(3,4), D_{b}(3,3,1)\right\}$, then the set $\mathcal{L}$ is not independent, a contradiction. If $F \in \mathcal{G}_{0}$, then $G$ contains a vertex of degree 3 adjacent to both ends of a 2-handle, a contradiction. If $F=D(4,4)$, then either $t=2$, in which case $\gamma_{t}^{d}(G)=3=(n-3) / 2$, or $t=5$, in which case $\gamma_{t}^{d}(G) \leq 5=(n-2) / 2$. Both cases produce a contradiction. This completes the proof of Lemma 23.

By Lemma 23, there is no 2-handle in $G$. Recall that by our earlier assumptions, $n \geq 8$ and the set $\mathcal{L}$ is an independent set. Further, $G$ does not contain a path on six vertices each internal vertex of which has degree 2 in $G$ and whose end vertices are not adjacent. In particular, every 2-path in $G$ has order at most 3 .

Lemma 24 If $G$ contains a 4-cycle, then $G=B_{8}$.

Proof of Lemma 24. Suppose that $G$ contains a 4-cycle uvwxu. Renaming vertices if necessary, we may assume that $u$ and $w$ are large vertices of $G$ (and so $v$ and $x$ are small vertices). We now consider the connected subgraph $G^{\prime}=G-v$ of $G$ that satisfies $\delta\left(G^{\prime}\right) \geq 2$. Let $G^{\prime}$ have order $n^{\prime}$, and so $n^{\prime}=n-1 \geq 7$. By Observation $18, G^{\prime} \in \mathcal{B} \cup \mathcal{C} \cup \mathcal{D} \cup \mathcal{D}_{b} \cup \mathcal{G}$ or $\gamma_{t}^{d}\left(G^{\prime}\right) \leq\left(n^{\prime}-2\right) / 2$. Every DTD-set in $G^{\prime}$ is also a DTD-set in $G$, implying that $\gamma_{t}^{d}(G) \leq \gamma_{t}^{d}\left(G^{\prime}\right)$. If $\gamma_{t}^{d}\left(G^{\prime}\right) \leq\left(n^{\prime}-1\right) / 2$, then $\gamma_{t}^{d}(G) \leq$ $(n-2) / 2$, a contradiction. Hence, $\gamma_{t}^{d}\left(G^{\prime}\right) \geq n^{\prime} / 2$. If $G^{\prime}$ is a cycle, then $G$ would contain a 2-path of order at least 4 , a contradiction. Hence by Observation $7, G^{\prime} \in\left\{B_{3}, D_{b}(4,4), D_{b}(3,4,1), D_{b}(3,3,2)\right\}$. If $G^{\prime} \in\left\{D_{b}(4,4), D_{b}(3,4,1), D_{b}(3,3,2)\right\}$, then $G$ contains a 2-handle, a contradiction. Hence, $G^{\prime}=B_{3}$, implying that $G=B_{8}$.

By Lemma 24, we may assume that $G$ contains no 4-cycle, for otherwise the desired result follows.

Lemma 25 There is no 5-cycle in $G$.

Proof of Lemma 24. Suppose to the contrary that $G$ contains a 5-cycle uvwxyu. Renaming vertices if necessary, we may assume that $u$ and $w$ are large vertices of $G$ (and so $v, x$ and $y$ are small vertices). We now consider the connected subgraph $G^{\prime}=G-v$ of $G$ that satisfies $\delta\left(G^{\prime}\right) \geq 2$. Let $G^{\prime}$ have order $n^{\prime}$, and so $n^{\prime}=n-1 \geq 7$. By Observation $18 G^{\prime} \in \mathcal{B} \cup \mathcal{C} \cup \mathcal{D} \cup \mathcal{D}_{b} \cup \mathcal{G}$ or $\gamma_{t}^{d}\left(G^{\prime}\right) \leq\left(n^{\prime}-2\right) / 2$. 
Every DTD-set in $G^{\prime}$ is also a DTD-set in $G$, implying that $\gamma_{t}^{d}(G) \leq \gamma_{t}^{d}\left(G^{\prime}\right)$. If $\gamma_{t}^{d}\left(G^{\prime}\right) \leq\left(n^{\prime}-1\right) / 2$, then $\gamma_{t}^{d}(G) \leq(n-2) / 2$, a contradiction. Hence, $\gamma_{t}^{d}\left(G^{\prime}\right) \geq n^{\prime} / 2$. By Observation 7 and since every 2-path in $G$ has order at most $3, G^{\prime} \in\left\{C_{7}, B_{3}, D_{b}(4,4), D_{b}(3,4,1), D_{b}(3,3,2)\right\}$. We note that uyxw is an induced path in $G^{\prime}$ where $x$ and $y$ have degree 2 in $G^{\prime}$. This implies that $G^{\prime} \notin\left\{D_{b}(4,4), D_{b}(3,4,1)\right\}$. If $G^{\prime}=C_{7}$, then $\gamma_{t}^{d}(G) \leq|\{u, v, w\}|=(n-2) / 2$, a contradiction. If $G^{\prime}=D_{b}(3,3,2)$, then $u$ and $w$ are necessarily the two large vertices in $G^{\prime}$. But then $G$ contains a 2-handle, a contradiction. If $G^{\prime}=B_{3}$, then $G$ contains a 4 -cycle, a contradiction.

By Lemma 25 and our assumptions to date, we may assume that a shortest cycle in $G$ has length at least 6 ; that is, $G$ has girth at least 6 . Recall that every 2-path in $G$ has order at most 3 .

Lemma 26 If $G$ has a 2-path of order 3 , then $G=B_{6}$.

Proof of Lemma 26. Let $P: v_{1} v_{2} v_{3}$ be a 2-path in $G$ and let $u$ and $v$ be the large vertices adjacent to $v_{1}$ and $v_{3}$, respectively.

Claim 13 If $u$ and $v$ do not have a common neighbor, then $G=B_{6}$.

Proof of Claim 13. Suppose that $u$ and $v$ do not have a common neighbor. We show that $G=B_{6}$. Let $N_{u}^{1}$ and $N_{u}^{2}$ be the set of vertices at distance 1 and 2, respectively, from $u$ in $G-V(P)$. Let $N_{v}^{1}$ and $N_{v}^{2}$ be defined analogously. By assumption, $N_{u}^{1} \cap N_{v}^{1}=\emptyset$. Every neighbor of a large vertex is a small vertex. In particular, every vertex in $N_{u} \cup N_{v}$ has degree 2 . Let $G^{\prime}$ be the graph of order $n^{\prime}=n-4$ obtained from $G-(V(P) \cup\{u\})$ by joining the vertices in $N_{u}^{1}$ to the vertex $v$. Since $G$ is an edge-minimal graph, so too is the graph $G^{\prime}$. Since $\left|N_{u}^{1}\right| \geq 2$ and $\left|N_{v}^{1}\right| \geq 2$, we note that $d_{G^{\prime}}(v)=\left|N_{u}^{1}\right|+\left|N_{v}^{1}\right| \geq 4$. Hence, $G^{\prime}$ is not a cycle and $G^{\prime}$ contains a vertex of degree at least 4 . Therefore by the induction hypothesis, either $G^{\prime} \in\left\{B_{4}, B_{8}, B_{9}, B_{10}\right\} \cup \mathcal{D} \cup \mathcal{G}$ or $\gamma_{t}^{d}\left(G^{\prime}\right) \leq\left(n^{\prime}-2\right) / 2$.

We show next that $\gamma_{t}^{d}\left(G^{\prime}\right) \geq\left(n^{\prime}-1\right) / 2$. Suppose to the contrary that $\gamma_{t}^{d}\left(G^{\prime}\right) \leq\left(n^{\prime}-2\right) / 2$. Let $S^{\prime}$ be a $\gamma_{t}^{d}\left(G^{\prime}\right)$-set. By construction, $N_{G^{\prime}}(v)=N_{u}^{1} \cup N_{v}^{1}$. Suppose that $S^{\prime}$ totally dominates the vertex $v$. Thus, $S^{\prime}$ contains at least one vertex in $N_{u}^{1} \cup N_{v}^{1}$. If $S^{\prime}$ contains a vertex in $N_{u}^{1}$ and a vertex in $N_{v}^{1}$, let $S=S^{\prime} \cup\{u, v\}$. If $S^{\prime}$ contains a vertex of $N_{v}^{1}$ and no vertex of $N_{u}^{1}$, let $S=S^{\prime} \cup\left\{u, v_{1}\right\}$. If $S^{\prime}$ contains no vertex of $N_{v}^{1}$ and $v \in S^{\prime}$, let $S=S^{\prime} \cup\left\{u, v_{3}\right\}$. If $S^{\prime}$ contains no vertex of $N_{v}^{1}$ and $v \notin S^{\prime}$, let $S=S^{\prime} \cup\left\{v, v_{3}\right\}$. In all four cases, the set $S$ is a DTD-set of $G$ and $|S|=\left|S^{\prime}\right|+2$, implying that $\gamma_{t}^{d}(G) \leq|S|=\left|S^{\prime}\right|+2=\left(n^{\prime}-2\right) / 2+2=(n-2) / 2$, a contradiction. Hence, $S^{\prime}$ does not totally dominates the vertex $v$.

Since $S^{\prime}$ contains no vertex in $N_{u}^{1} \cup N_{v}^{1}$, the set $S^{\prime}$ disjunctively dominates $v$ and therefore contains at least two vertices in $N_{u}^{2} \cup N_{v}^{2}$. If $v \in S^{\prime}$ and $S^{\prime}$ contains no vertex in $N_{u}^{2}$, let $S=S^{\prime} \cup\left\{u, v_{1}\right\}$. If $v \in S^{\prime}$ and $S^{\prime}$ contains exactly one vertex in $N_{u}^{2}$, let $S=S^{\prime} \cup\left\{u, v_{2}\right\}$. If $v \in S^{\prime}$ and $S^{\prime}$ contains at least two vertices in $N_{u}^{2}$, let $S=S^{\prime} \cup\left\{u, v_{3}\right\}$. If $v \notin S^{\prime}$ and $S^{\prime}$ contains no vertex in $N_{u}^{2}$, let $S=S^{\prime} \cup\left\{v_{1}, v_{2}\right\}$. If $v \notin S^{\prime}$ and $S^{\prime}$ contains exactly one vertex in $N_{u}^{2}$, let $S=S^{\prime} \cup\left\{u, v_{2}\right\}$. If $v \notin S^{\prime}$ and $S^{\prime}$ contains at least two vertices in $N_{u}^{2}$, let $S=S^{\prime} \cup\left\{v_{2}, v_{3}\right\}$. In all six cases, the set $S$ is a DTD-set of $G$ and $|S|=\left|S^{\prime}\right|+2$, implying that $\gamma_{t}^{d}(G) \leq|S|=\left|S^{\prime}\right|+2=\left(n^{\prime}-2\right) / 2+2=(n-2) / 2$, a contradiction.Therefore, $\gamma_{t}^{d}\left(G^{\prime}\right) \geq\left(n^{\prime}-1\right) / 2$, implying that $G^{\prime} \in\left\{B_{4}, B_{8}, B_{9}, B_{10}\right\} \cup \mathcal{D} \cup \mathcal{G}$.

Since $\mathcal{L}$ is an independent set in $G$, by construction the set of large vertices in $G^{\prime}$, namely the set $\mathcal{L} \backslash\{u\}$, form an independent set in $G^{\prime}$. If $G^{\prime} \in \mathcal{G}$, then since $v$ has degree at least 4 in $G^{\prime}$ and since the large vertices in $G^{\prime}$ are independent, $G^{\prime} \in \mathcal{G}_{0} \cup \mathcal{G}_{1}$ (and $G^{\prime}$ consists only of type-1 units). But then the graph $G$ would contain a 2-handle, a contradiction. Hence, $G^{\prime} \notin \mathcal{G}$. 
If $G^{\prime}=B_{4}$, then $v$ is one of the two vertices of degree 4 in $G^{\prime}$, implying that the graph $G$ contains a 2-path on at least four vertices as well as a 4-cycle, a contradiction. If $G^{\prime} \in\left\{B_{8}, B_{9}, B_{10}\right\}$, then $v$ is the vertex of degree 4 in $G^{\prime}$. If $G^{\prime} \in\left\{B_{9}, B_{10}\right\}$, then $G$ would contain a 2-handle, a contradiction. If $G^{\prime}=B_{8}$, then since $G$ contains no 4-cycles, there is only one way to reconstruct the graph $G$ from $G^{\prime}$. In this case, $n=13$ and the four large vertices in $G$ form a DTD-set of $G$, implying that $\gamma_{t}^{d}(G) \leq 4=$ $(n-5) / 2$, a contradiction. Hence, $G^{\prime} \notin\left\{B_{4}, B_{8}, B_{9}, B_{10}\right\}$. Therefore, $G \in \mathcal{D}$. But then $\mathcal{L}=\{u, v\}$ and $\left\{u, v_{1}, v_{3}, v\right\}$ is a DTD-set of $G$, implying that $\gamma_{t}^{d}(G) \leq 4$. If $n \geq 10$, then $\gamma_{t}^{d}(G) \leq(n-2) / 2$, a contradiction. Hence, $n=9$, implying that $G^{\prime}=D(3,3)$ and $G=B_{6}$. This completes the proof of Claim 13

By Claim 13, we may assume that $u$ and $v$ have a common neighbor, $w$ say, for otherwise $G=B_{6}$, and the desired result follows. Since $G$ has no 4-cycle, the vertex $w$ is the only common neighbor of $u$ and $v$. Let $G^{\prime}$ be the graph obtained from $G-(V(P) \cup\{w\})$ by adding the edge $e=u v$. Then, $G^{\prime}$ is a connected graph with $\delta\left(G^{\prime}\right) \geq 2$. Let $G^{\prime}$ have order $n^{\prime}$, and so $n^{\prime}=n-4$.

We show that $G^{\prime}-e$ is an edge-minimal graph. Suppose to the contrary that $G^{\prime}-e=G-(V(P) \cup\{w\})$ is not an edge-minimal graph. Then $G^{\prime}-e$ is disconnected or at least one of $u$ or $v$ has degree 1 in $G^{\prime}-e$. This implies that the graph $G^{\prime}$ is an edge-minimal graph. Then, $\gamma_{t}^{d}\left(G^{\prime}\right) \leq\left(n^{\prime}-2\right) / 2$ or $G^{\prime} \in \mathcal{B} \cup \mathcal{C} \cup \mathcal{D} \cup \mathcal{D}_{b} \cup \mathcal{G}$. Let $S^{\prime}$ be a $\gamma_{t}^{d}\left(G^{\prime}\right)$-set. Suppose $\gamma_{t}^{d}\left(G^{\prime}\right) \leq\left(n^{\prime}-2\right) / 2$. If $u$ or $v$ belong to $S^{\prime}$, let $S=S^{\prime} \cup\{u, v, w\}$. Suppose that neither $u$ nor $v$ belong to $S^{\prime}$. If both $u$ and $v$ have a neighbor in $S^{\prime}$, let $S=S^{\prime} \cup\{u, v\}$. If $u$ has a neighbor in $S^{\prime}$, let $S=S^{\prime} \cup\left\{v_{1}, w\right\}$. If $v$ has a neighbor in $S^{\prime}$, let $S=S^{\prime} \cup\left\{v_{3}, w\right\}$. If neither $u$ nor $v$ have a neighbor in $S^{\prime}$, let $S=S^{\prime} \cup\{u, v\}$. In all the above cases, the set $S$ is a DTD-set of $G$ and $|S| \leq\left|S^{\prime}\right|-2$, implying that $\gamma_{t}^{d}(G) \leq|S| \leq\left(n^{\prime}-2\right) / 2=(n-2) / 2$, a contradiction. Hence, $G^{\prime} \in \mathcal{B} \cup \mathcal{C} \cup \mathcal{D} \cup \mathcal{D}_{b} \cup \mathcal{G}$.

If $G^{\prime}$ is a cycle, then since $G$ has girth at least 6 and every 2-path in $G$ has order at most 3, we deduce that $G^{\prime}=C_{5}$. But then the graph $G$ is determined. In this case, $n=9$ and $\{u, v, w\}$ is a DTD-set of $G$, and so $\gamma_{t}^{d}(G)=3=(n-3) / 2$, a contradiction. Hence, $G^{\prime}$ is not a cycle, implying that $\gamma_{t}^{d}\left(G^{\prime}\right) \leq n^{\prime} / 2$. If $e$ is a good-edge of $G^{\prime}$, then choosing $S^{\prime}$ to be a $\gamma_{t}^{d}\left(G^{\prime}\right)$-set that contains both $u$ and $v$, the set $S^{\prime} \cup\{w\}$ is a DTD-set of $G$, implying that $\gamma_{t}^{d}(G) \leq\left|S^{\prime}\right|+1 \leq n^{\prime} / 2+1=(n-2) / 2$, a contradiction. Hence, $e$ is a bad-edge of $G^{\prime}$. However applying Observation 10 to the graph $G^{\prime}$ and the bad-edge $e$ of $G^{\prime}$, the graph $G$ necessarily contains a 3-cycle or a 4-cycle, a contradiction. Therefore, $G^{\prime}-e=G-(V(P) \cup\{w\})$ is an edge-minimal graph.

Thus, $\gamma_{t}^{d}\left(G^{\prime}-e\right) \leq\left(n^{\prime}-2\right) / 2$ or $G^{\prime}-e \in \mathcal{B} \cup \mathcal{C} \cup \mathcal{D} \cup \mathcal{D}_{b} \cup \mathcal{G}$. Let $S^{\prime}$ be a $\gamma_{t}^{d}\left(G^{\prime}-e\right)$-set. If $\gamma_{t}^{d}\left(G^{\prime}-e\right) \leq\left(n^{\prime}-2\right) / 2$, then $S^{\prime} \cup\{u, v\}$ is a DTD-set of $G$, implying that $\gamma_{t}^{d}(G) \leq|S| \leq\left(n^{\prime}-2\right) / 2=$ $(n-2) / 2$, a contradiction. Hence, $G^{\prime}-e \in \mathcal{B} \cup \mathcal{C} \cup \mathcal{D} \cup \mathcal{D}_{b} \cup \mathcal{G}$. Since both $u$ and $v$ have degree at least 2 in $G^{\prime}-e$, we note that $G^{\prime} \notin \mathcal{D}$. If $G^{\prime}-e \in \mathcal{D}_{b} \cup \mathcal{G}$, then $G$ would contain a 3 -cycle or a 4-cycle, a contradiction. If $G^{\prime}-e$ is a cycle, then since $G$ has girth at least 6 and every 2-path in $G$ has order at most 3 , we note that $G^{\prime} \in\left\{C_{6}, C_{7}\right\}$ and $u$ and $v$ are at distance 3 apart on the cycle. But then the graph $G$ is determined. In this case, $n \in\{10,11\}$ and $\{u, v, w\}$ is a DTD-set of $G$, and so $\gamma_{t}^{d}(G)=3 \leq(n-4) / 2$, a contradiction. If $G^{\prime}-e \in \mathcal{B}$, then since $G$ has girth at least $6, G^{\prime}-e=B_{6}$ and $u$ and $v$ are the two vertices of degree 3 in $G^{\prime}-e$. But then $n=13$ and $\{u, v, w\}$ is a DTD-set of $G$, and so $\gamma_{t}^{d}(G)=3=(n-7) / 2$, a contradiction. This completes the proof of Lemma 26 .

By Lemma 26, if $G$ has a 2-path of order 3, then $G=B_{6}$. Hence we may assume that every 2-path in $G$ has order 1 or 2 . Thus every small vertex (of degree 2 ) has either two large neighbors or one large neighbor and one small neighbor depending on whether it belongs to a 2-path of order 1 or a 2-path of 
order 2 , respectively.

Let $\mathcal{S}=\left(\mathcal{S}_{1}, \mathcal{S}_{2}\right)$ be a weak partition of $\mathcal{S}$ (a partition where some of the sets may be empty), where $\mathcal{S}_{1}$ is the set of small vertices with two large neighbors and $\mathcal{S}_{2}$ is the set of small vertices with exactly one large neighbor. We note that $G\left[\mathcal{S}_{2}\right]$ consists of the disjoint union of paths of order 2.

Let $\mathcal{L}=\left(\mathcal{L}_{0}, \mathcal{L}_{1}, \mathcal{L}_{2}\right)$ be a weak partition of the large vertices $\mathcal{L}$, where $\mathcal{L}_{0}, \mathcal{L}_{1}$ and $\mathcal{L}_{2}$ are the set of large vertices adjacent to zero, one and at least two vertices in $\mathcal{S}_{1}$, respectively, respectively. Let $\mathcal{S}_{1,1}$ be the set of vertices in $\mathcal{S}_{1}$ with both neighbors in $\mathcal{L}_{1}$ and let $\mathcal{S}_{1,2}$ be the set of vertices in $\mathcal{S}_{1}$ with exactly one neighbor in $\mathcal{L}_{1}$ (and the other neighbor in $\mathcal{L}_{2}$ ). Further, for $i \in\{1,2\}$ let $\mathcal{L}_{1, i}$ be the set of vertices in $\mathcal{L}_{1}$ adjacent to a vertex in $\mathcal{S}_{1, i}$. Thus, $\left(\mathcal{L}_{1,1}, \mathcal{L}_{1,2}\right)$ is a partition of $\mathcal{L}_{1}$.

Let $|\mathcal{S}|=s,\left|\mathcal{S}_{1}\right|=s_{1}$ and $\left|\mathcal{S}_{2}\right|=2 s_{2}$, and so $s=s_{1}+2 s_{2}$. Let $\left|\mathcal{S}_{1,1}\right|=s_{1,1}$ and $\left|\mathcal{S}_{1,2}\right|=s_{1,2}$, and so $s_{1} \geq s_{1,1}+s_{1,2}$. Let $|\mathcal{L}|=\ell,\left|\mathcal{L}_{0}\right|=\ell_{0},\left|\mathcal{L}_{1}\right|=\ell_{1}$, and $\left|\mathcal{L}_{2}\right|=\ell_{2}$, and so $\ell=\ell_{0}+\ell_{1}+\ell_{2}$. Let $\left|\mathcal{L}_{1,1}\right|=\ell_{1,1}$ and $\left|\mathcal{L}_{1,2}\right|=\ell_{1,2}$, and so $\ell_{1}=\ell_{1,1}+\ell_{1,2}$ and

$$
\ell=\ell_{0}+\ell_{1,1}+\ell_{1,2}+\ell_{2} .
$$

The subgraph $G\left[\mathcal{L}_{1,1} \cup \mathcal{S}_{1,1}\right]$ induced by $\mathcal{L}_{1,1} \cup \mathcal{S}_{1,1}$ consists of a disjoint union of paths $P_{3}$ on three vertices (where the internal vertices of these paths form the set $\mathcal{S}_{1,1}$ ), while $G\left[\mathcal{L}_{1,2} \cup \mathcal{S}_{1,2}\right]$ consists of a disjoint union of paths $P_{2}$ on two vertices (where each path contains one vertex of $\mathcal{L}_{1,2}$ and one vertex of $\mathcal{S}_{1,2}$.) Therefore,

$$
\ell_{1,1}=2 s_{1,1} \quad \text { and } \quad \ell_{1,2}=s_{1,2}
$$

implying that

$$
s_{1} \geq \frac{1}{2} \ell_{1,1}+\ell_{1,2}
$$

Counting the edge joining the large vertices to the ends of 2-paths of order 2, this sum is exactly $2 s_{2}$ and at least $3 \ell_{0}+2 \ell_{1}+\ell_{2}$. Thus,

$$
2 s_{2} \geq 3 \ell_{0}+2 \ell_{1,1}+2 \ell_{1,2}+\ell_{2}
$$

By (1), (2) and (3) we therefore have that

$$
\begin{aligned}
n & =\ell+s_{1}+2 s_{2} \\
& \geq 4 \ell_{0}+\frac{7}{2} \ell_{1,1}+4 \ell_{1,2}+2 \ell_{2} \\
& \geq 4 \ell_{0}+3 \ell_{1,1}+4 \ell_{1,2}+2 \ell_{2} \\
& =2 \ell+2 \ell_{0}+\ell_{1,1}+2 \ell_{1,2},
\end{aligned}
$$

or, equivalently,

$$
\frac{n}{2} \geq \ell+\ell_{0}+\frac{1}{2} \ell_{1,1}+\ell_{1,2} .
$$

For each vertex $v \in \mathcal{L}_{0}$, let $v^{\prime}$ be an arbitrary neighbor of $v$. Let $D^{\prime}=\bigcup\left\{v^{\prime}\right\}$, where the union is taken over all vertices $v \in \mathcal{L}_{0}$. Then, $\left|D^{\prime}\right|=\ell_{0}$. We now consider the set

$$
D=\mathcal{L} \cup D^{\prime} \cup \mathcal{S}_{1,1} \cup \mathcal{S}_{1,2} .
$$


Every vertex in $G$ is totally dominated by the set $D$, except possibly for vertices in $\mathcal{L}_{2}$ which are disjunctively dominated by $D$ (since each vertex in $\mathcal{L}_{2}$ is at distance 2 from at least two vertices in $\mathcal{L}$ ). The set $D$ is therefore a DTD-set of $G$, implying by (4) that

$$
\begin{aligned}
\gamma_{t}^{d}(G) & \leq|D| \\
& =\ell+\ell_{0}+s_{1,1}+s_{1,2} \\
& =\ell+\ell_{0}+\frac{1}{2} \ell_{1,1}+\ell_{1,2} \\
& \leq \frac{n}{2} .
\end{aligned}
$$

Lemma 27 Every 2-path has order 2; that is, $\mathcal{L}=\mathcal{L}_{0}$.

Proof of Lemma 27. If $\mathcal{L}_{1,2} \neq \emptyset$, then removing from $D$ an arbitrary vertex that belongs to the set $\mathcal{L}_{1,2}$ produces a DTD-set of cardinality $|D|-1$, implying that $\gamma_{t}^{d}(G) \leq(n-2) / 2$, a contradiction. Hence, $\mathcal{L}_{1,2}=\emptyset$. This in turn implies that $\mathcal{S}_{1,2}=\emptyset$. Thus, $\ell_{1,2}=s_{1,2}=0$ and Inequality 2 simplifies to $s_{1} \geq \frac{1}{2} \ell_{1,1}$.

If $\mathcal{L}_{2} \neq \emptyset$, then there are at least two vertices in $\mathcal{S}_{1}$ that do not belong to $\mathcal{S}_{1,1}$, and so $s_{1} \geq 2+\frac{1}{2} \ell_{1,1}$. But then Inequality (4) can be strengthened to $\frac{n}{2} \geq \ell+\ell_{0}+\frac{1}{2} \ell_{1,1}+1$, implying that $\gamma_{t}^{d}(G) \leq|D| \leq n / 2-1$, a contradiction. Hence, $\mathcal{L}_{2}=\emptyset$. Thus, $\ell_{2}=0$ and $s_{1}=\frac{1}{2} \ell_{1,1}$.

Suppose that $\mathcal{L}_{1} \neq \emptyset$. Then, by our earlier observations, $\mathcal{L}_{1}=\mathcal{L}_{1,1}$. As observed earlier, $\ell_{1,1}$ is even and the subgraph $G\left[\mathcal{L}_{1,1} \cup \mathcal{S}_{1,1}\right]$ induced by $\mathcal{L}_{1,1} \cup \mathcal{S}_{1,1}$ consists of a disjoint union of paths $P_{3}$ with the internal vertices of these paths forming the set $\mathcal{S}_{1,1}$. Removing from $D$ an arbitrary vertex that belongs to the set $\mathcal{L}_{1,1}$ produces a DTD-set of cardinality $|D|-1$, implying that $\gamma_{t}^{d}(G) \leq(n-2) / 2$, a contradiction. Hence, $\mathcal{L}_{1}=\emptyset$, implying that $\mathcal{L}=\mathcal{L}_{0}$. This completes the proof of Lemma 27 .

By Lemma 27, every 2-path in $G$ has order 2, implying that $\mathcal{L}=\mathcal{L}_{0}$ and $D=\mathcal{L}_{0} \cup D^{\prime}$.

Lemma 28 There is no 6-cycle in $G$.

Proof of Lemma 28. Suppose to the contrary that $G$ contains a 6-cycle $C: v_{1} v_{2} v_{3} v_{4} v_{5} v_{6} v_{1}$. Renaming vertices if necessary, we may assume that $v_{1}$ and $v_{4}$ are large vertices. Recall that for each vertex $v \in \mathcal{L}_{0}$, we let $v^{\prime}$ be an arbitrary neighbor of $v$ and the set $D^{\prime}=\bigcup\left\{v^{\prime}\right\}$, where the union is taken over all vertices $v \in \mathcal{L}_{0}$. We now remove from $D$ the vertex $v_{1}$ and the two vertices $v_{1}^{\prime}$ and $v_{4}^{\prime}$ (that belong to $D^{\prime}$ ) and we replace them with the two neighbors of $v_{1}$ on $C$ (namely, the vertices $v_{2}$ and $v_{6}$ ). The resulting set is a DTD-set of cardinality $|D|-1$, implying that $\gamma_{t}^{d}(G) \leq(n-2) / 2$, a contradiction.

By Lemma $28 G$ contains no 6-cycle, implying that the girth of $G$ is at least 7 . This in turn implies that $|\mathcal{L}| \geq 4$. We now choose an arbitrary vertex $v \in \mathcal{L}$. Let $v_{1}, v_{2}, \ldots, v_{q}$ be the vertices in $\mathcal{L}$ at distance 3 from $v$. Since $G$ contains no 6 -cycle, we note that $q=d_{G}(v)$. For $i=1,2, \ldots, q$, let $v_{i} a_{i} b_{i} v$ be a path in $G$ (and so, $a_{i} b_{i}$ is a 2-path in $G$ of order 2 whose one end $a_{i}$ is adjacent to $v_{i}$ and whose other end $b_{i}$ is adjacent to $v$ ). If we now choose the vertex $v_{i}^{\prime}$ to be the vertex $a_{i}$ for each $i=1,2, \ldots, q$, then the vertex $v$ can be removed from $D$ to produce a DTD-set of cardinality $|D|-1$, implying that $\gamma_{t}^{d}(G) \leq(n-2) / 2$, a contradiction. This completes the proof of Theorem 16

\subsection{Proof of Theorem 4}

Let $G$ be a connected graph of order $n \geq 8$ with $\delta(G) \geq 2$. Since $\gamma_{t}^{d}(G)$ cannot increase if edges are added, it follows from Theorem 16 and Observation 7 that $\gamma_{t}^{d}(G) \leq n / 2$. Further, suppose $\gamma_{t}^{d}(G)=n / 2$. We produce a $\frac{1}{2}$-minimal graph $G^{\prime}$ from $G$ by removing edges if necessary so that $G^{\prime}$ satisfies $\gamma_{t}^{d}\left(G^{\prime}\right)=$ 
$n / 2$. By Theorem 16 and Observation 7. $G^{\prime} \in\left\{B_{3}, D_{b}(4,4), D_{b}(3,4,1), D_{b}(3,3,2), C_{8}, C_{12}\right\}$. In all cases it can be readily checked that $G=G^{\prime}$ or $G^{\prime} \in \mathcal{F}$ where $\mathcal{F}$ is the family of graphs shown in Fig. 5

\subsection{Proof of Theorem 5}

Let $G$ be a connected graph of order $n \geq 18$ with $\delta(G) \geq 2$. Since $\gamma_{t}^{d}(G)$ cannot increase if edges are added, it follows from Theorem 16 and Observation 7 that $\gamma_{t}^{d}(G) \leq(n-1) / 2$. Further, suppose $n \geq 18$ and $\gamma_{t}^{d}(G)=(n-1) / 2$. We produce a $\frac{1}{2}$-minimal graph $G^{\prime}$ from $G$ by removing edges if necessary so that $G^{\prime}$ satisfies $\gamma_{t}^{d}\left(G^{\prime}\right)=(n-1) / 2$. Since $n \geq 18, G^{\prime} \in \mathcal{G}$ by Observation 7 It can readily be checked that $G=G^{\prime}$ or $G \in \mathcal{H}$ where $\mathcal{H}$ is the family of graphs constructed in Section 1.2

\section{References}

D. Archdeacon, J. Ellis-Monaghan, D. Fischer, D. Froncek, P. C. B. Lam, S. Seager, B. Wei, and R. Yuster. Some remarks on domination. J. Graph Theory, 46:207-210, 2004.

S. Bornholdt and H. G. Schuster. Handbook of Graphs and Networks: From the Genome to the Internet. Wiley-Vch GmbH \& Co. KGaA, Weinheim, 2003.

R. C. Brigham, J. R. Carrington, and R. P. Vitray. Connected graphs with maximum total domination number. J. Combin. Comput. Combin. Math., 34(111):81-96, 2000.

F. Chung. Graph theory in the information age. Notices Amer. Math. Society, 57:726-732, 2010.

V. Chvátal and C. McDiarmid. Small transversals in hypergraphs. Combinatorica, 12:19-26, 1992.

E. J. Cockayne, R. M. Dawes, and S. T. Hedetniemi. Total domination in graphs. Networks, 10:211-219, 1980.

W. Goddard, M. A. Henning, and C. A. McPillan. The disjunctive domination number of a graph. Quaestiones Mathematicae, 37(4):547-561, 2014.

T. W. Haynes, S. T. Hedetniemi, and P. J. Slater. Fundamentals of domination in graphs. Monographs and textbooks in pure and applied mathematics. Marcel Dekker, 1998.

M. A. Henning. Graphs with large total domination number. J. Graph Theory, 35:21-45, 2000.

M. A. Henning. Recent results on total domination in graphs: A survey. Discrete Math., 309:32-63, 2009.

M. A. Henning and V. Naicker. Graphs with large disjunctive total domination number, http://arxiv.org/pdf/1409.1681v2.pdf.

M. A. Henning and V. Naicker. The disjunctive total domination number of a graph. J. Comb. Optim., 2014.

M. A. Henning and A. Yeo. Total domination in graphs. Springer Monographs in Mathematics. Springer, 2013.

Z. Tuza. Covering all cliques of a graph. Discrete Math., 86:117-126, 1990. 
